\begin{abstract}
Photoconductive antennas are promising sources of terahertz radiation that is widely used for spectroscopy, characterization, and imaging of biological objects, deep space studies, scanning of surfaces, and detection of potentially hazardous substances. These antennas are compact and allow for generation of both ultrabroadband pulses and tunable continuous wave terahertz signals at room temperatures, with no need for highpower optical sources. However, such antennas have relatively low energy conversion efficiency of femtosecond laser pulses or two close pump wavelengths (photomixers) into the pulsed and continuous terahertz radiation, correspondingly. Recently, an approach to solving this problem that involves known methods of nanophotonics applied to terahertz photoconductive antennas and photomixers has been proposed. This approach comprises the use of optical nanoantennas for enhancing the absorption of pump laser radiation in the antenna gap, reducing the lifetime of photoexcited carriers, and improving the antenna thermal efficiency. This Review is intended to systematize the main results obtained by researchers in this promising field of hybrid optical-to-terahertz photoconductive antennas and photomixers. We summarize the main results on hybrid $\mathrm{THz}$ antennas, compare the approaches to their implementation, and offer further perspectives of their development including an application of all-dielectric nanoantennas instead of plasmonic ones.
\end{abstract}

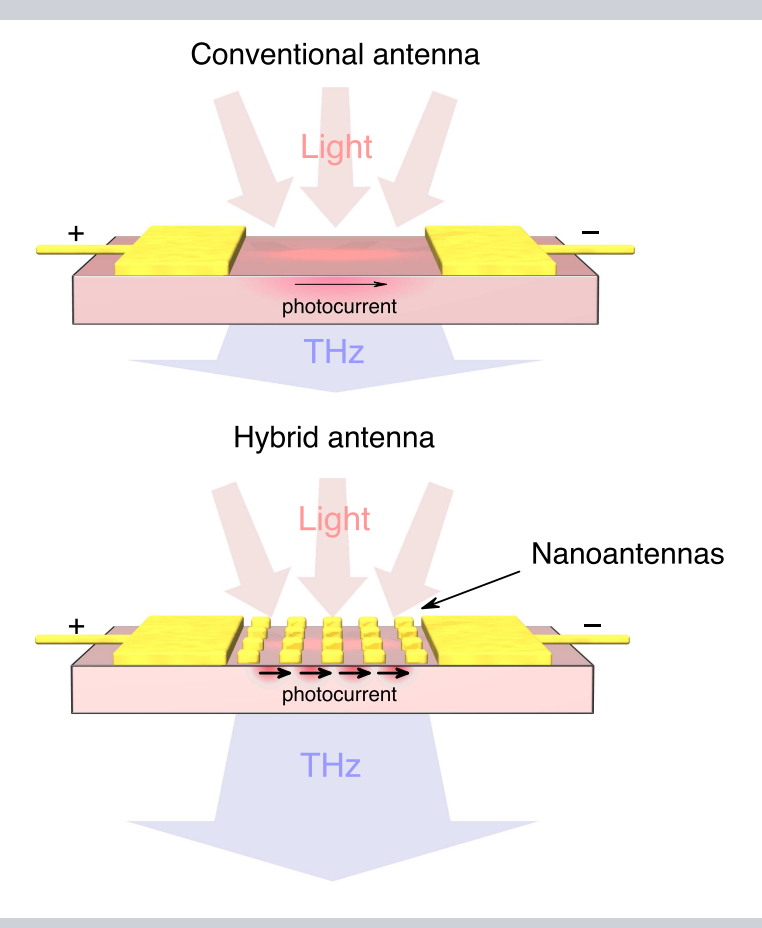

\title{
Enhancement of Terahertz Photoconductive Antenna Operation by Optical Nanoantennas
}

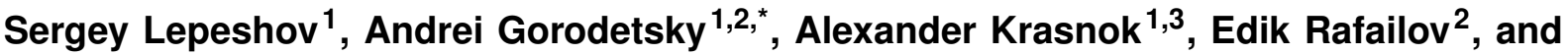 \\ Pavel Belov ${ }^{1}$
}

\section{Introduction}

The terahertz (THz) spectral range of electromagnetic waves $(0.1-10 \mathrm{THz})$, lying between the microwave and optical regions, is of great interest, primarily due to the fact that in this band of the electromagnetic spectrum reside the frequencies of some elementary excitations in semiconductors and dielectrics [1-12], as well as rotational and vibrational resonances of complex, including biological, molecules [13-21]. As a result, THz waves have a tremendous number of applications in areas ranging from the detection of dangerous or illicit substances [6,13,22-27] and biological sensing [28-33] to diagnosis and treatment of diseases in medicine [20,21,34-41]. Moreover, this frequency band is of special importance for spectroscopy in astrophysics [42-47] because many astrophysical objects emit in the $\mathrm{THz}$ range of the spectrum. In addition, the $\mathrm{THz}$ technology is promising for wireless communication sys- tems $[48,49]$ as a complementary technology to transmitters and receivers operating in high-frequency and microwave ranges, because it can potentially increase the speed of data transfer by hundreds of times [50-55]. Although, there are some reasonable hurdles, like strong absorption of $\mathrm{THz}$ waves by metals and water, on the way to the real life application of THz wireless technology to add bandwidth to mega- and gigahertz frequency devices [56-58], a significant progress has already been made [5,59-61].

Until relatively recently, the generation of coherent $\mathrm{THz}$ waves was an extremely difficult task. However, research in semiconductors [7, 8, 62-66], and in the interaction of short (compared with all relaxation times) optical pulses with semiconductor and nonlinear materials (photoconductivity effect $[67,68])$ stimulated the development of compact lowpower $\left(\sim 10^{-14} \mathrm{~J} /\right.$ pulse $) \mathrm{THz}$ radiation transmitters.

\footnotetext{
1 ITMO University, St. Petersburg, 197101, Russia

2 Aston Institute of Photonic Technologies, Aston University, B4 7ET, Birmingham, UK

${ }^{3}$ Department of Electrical and Computer Engineering, The University of Texas at Austin, Austin, Texas 78712, USA

* Corresponding author: e-mail: andrei@corp.ifmo.ru
} 


\section{Conventional antenna}

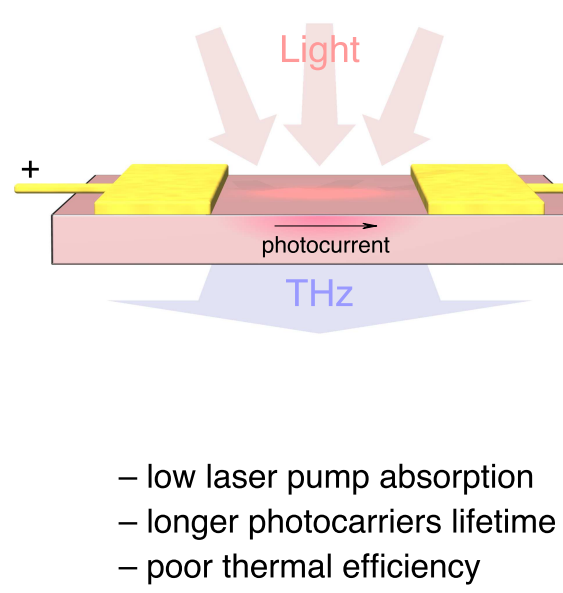

Hybrid antenna

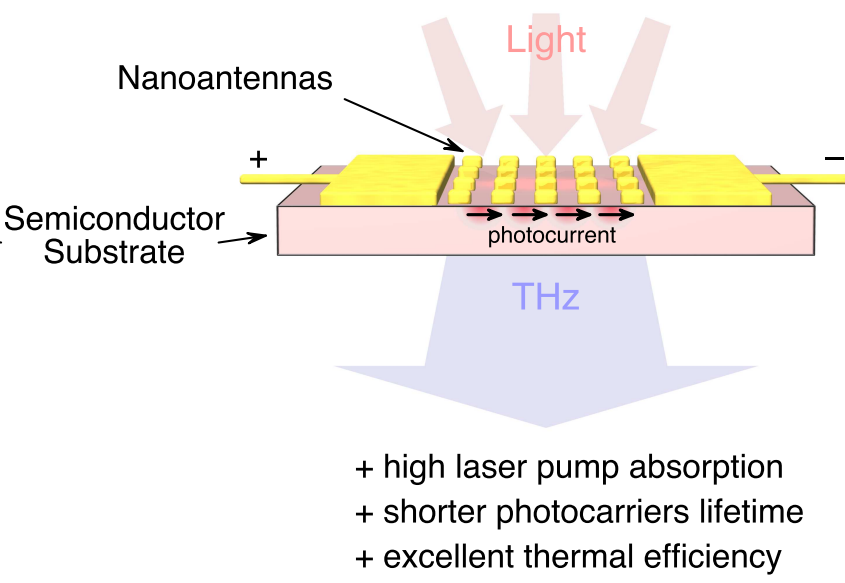

Figure 1 Illustration of the hybrid antenna advantages over conventional one.

Nowadays, the most common method for a low power $\mathrm{THz}$ generation by conversion of an optical pump involves the use of semiconductor structures with a surface conductivity $[69,70]$. Two or more conductive electrodes spaced by a certain gap are deposited onto a semiconductor surface (Figure 1, left side). The electrodes are biased by the external voltage of several V. Such structure is referred to as $\mathrm{THz}$ photoconductive antenna if pumped by femtosecond (fs) optical pulses, or $\mathrm{THz}$ photomixer in the case of continuous (CW) pump by two lasers operating at close wavelengths with a difference being in the THz spectral range. Upon excitation the gap between the electrodes with a fs-laser, the concentration of charge carriers increases sharply for a short period of time, and the THz pulse generation occurs. The duration of this pulse and its spectrum are determined mainly by the carriers lifetime in the semiconductor. In the case of $\mathrm{CW}$ pumping, the carriers concentration changes with the difference frequency of pump wavelengths, and CW $\mathrm{THz}$ signal is emitted. The efficiency of such $\mathrm{THz}$ sources is strongly limited by the amount of the optical energy of laser radiation absorbed in the gap of the photoconductive antenna. Usually, this amount is rather small, taking into account the high values of the semiconductor refractive index at optical frequencies, leading to a high reflection coefficient. Moreover, the effectiveness of conventional photoconductive antennas is limited by low drift velocities of the photoinduced charge carriers in semiconductor substrates [71-73] and material breakdown threshold [74].

It has been shown recently, that the efficiency of the $\mathrm{THz}$ pulse generation can be significantly increased by placing so-called optical nanoantennas (NAs) [75-84] into the photoconductive gap (Figure 1, right side) [69, 70, 74, 85-90]. As a result, one gets an antenna comprising a comprehensive photoconductive $\mathrm{THz}$ antenna and an array of optical NAs. Such system can be called a hybrid THz-optical photoconductive antenna. These antennas are the result of the application of latest advances in nanophotonics area to $\mathrm{THz}$ sources technology for the purpose of their efficiency enhancement. The efficiency of the entire hybrid antenna is determined by the efficiencies of $\mathrm{THz}$ photoconductive antenna and an optical NA array and is usually higher by at least one order of magnitude than the efficiency of conventional $\mathrm{THz}$ antenna. Besides the pump field enhancement in the semiconductor substrate, the plasmonic NAs improve the thermal stability of the $\mathrm{THz}$ photoconductive antenna due to the large thermal conductivity of metals. As for detectors, the use of hybrid photoconductive $\mathrm{THz}$ detectors with optically resonant nanoparticles in the photoconductive gap allows for the enhancement of the near-field $\mathrm{THz}$ imaging resolution up to $\lambda / 150$ [91]. This unprecedented resolution has become available due to the extremely high scattering provided by NAs into the semiconductor [i.e., low $(<200 \mathrm{C})$ temperature grown gallium arsenide (LT-GaAs)] detector surface layer and hence high enough concentration of carriers in the ultrathin semiconductor layer next to subwavelength detector aperture for evanescent $\mathrm{THz}$ field registration.

Another type of perspective $\mathrm{THz}$ detectors involves the use of plasmonic structures with resonances in $\mathrm{THz}$ frequencies [92-98], this technique can also be used for gaining $\mathrm{THz}$ spectrometers sensitivity through local field enhancement, however such devices wont fall within the scope of this Review.

This Review is intended to systematize all main results obtained by researchers in the field of hybrid optical-toterahertz photoconductive antennas and photomixers. First, we just briefly describe the main operating principles of $\mathrm{THz}$ generation in photoconductive antennas and photomixers pumped with fs-optical pulses and double-wavelength $\mathrm{CW}$ laser radiation, since there is a great number of excellent reviews on this subject (among them, we recommend the following Refs. [6, 50, 62, 99-101]). Second, we discuss the general principles of operation of optical NAs and the prospects they offer for $\mathrm{THz}$ emission. We describe the plas- 
mon monopole and dipole optical nanoantennas, two- and three-dimensional plasmonic gratings and features of their operation in hybrid $\mathrm{THz}$ antennas. Finally, we summarize and compare the main approaches to the implementation of the hybrid antennas, and give an outlook of further perspectives of their development including an application of all-dielectric NAs instead of plasmonic ones and the search for new materials.

\section{Principles of $\mathrm{THz}$ photoconductive antennas and photomixers}

\subsection{Methods of coherent $\mathrm{THz}$ generation}

One of the simplest, but perhaps not the most efficient, way to obtain the $\mathrm{THz}$ waves is by accelerated movement of the separated charge carriers in the surface field of a semiconductor under ultrashort light pulses $[7,67]$. One of the physical mechanisms that drive the carriers inside a semiconductor is the photo-Dember effect. This effect consists in the formation of the electric field (and consequently a charge dipole) in the illuminated semiconductor due to the difference of electrons and holes diffusion velocities [102] under the strong absorption of optical excitation, which leads to an effective charge separation in the direction perpendicular to the semiconductor surface [103].

Another possible way to generate $\mathrm{THz}$ waves - optical rectification - works when a second order nonlinear media obtains a non-linear polarization under an intense optical radiation. It results in a polarization repeating the shape of the optical pulse envelope [104-106] or in the case of four-wave mixing in third-order nonlinearity, such as in $\mathrm{THz}$ generation via two-color laser-plasmas [107, 108], though the latter requires higher pulse energies produced by chirped pulse amplification [109].

Apart from these well-established methods, novel approaches of nanophotonics to $\mathrm{THz}$ generation that employ an artificial electromagnetic medium (metamaterials and metasurfaces) [110-118], and new types of materials (graphene [119,120], carbon nanotubes [121,122], and threedimensional topological insulators such as $\mathrm{Bi}_{2} \mathrm{Se}_{3}$ [123]) have been recently proposed.

We should note here that the main competitors of photonic techniques for coherent $\mathrm{THz}$ radiation generation are all-electronic direct $\mathrm{THz}$ Quantum Cascade Lasers (QCLs) [124-126] and on-chip nonlinear semiconductor mixers of mid-IR QCL radiation [127, 128]. Monolithic design offers reasonable advantages over now table-top photonic setups, however, QCLs are extremely expensive in design and production, and direct $\mathrm{THz}$ QCLs operate only at cryogenic temperatures. In the scope of applications, QCLs will barely offer fine and continuous tunability, or generation of ultrabroadband $\mathrm{THz}$ signal, needed for spectroscopy. As a result, QCLs are geared mostly towards imaging, whereas photonic sources are especially demanded for spectroscopic applications. Development of antenna-based monolithic THz sources in conjunction with compact semiconductor lasers is also highly feasible [129]. (a)

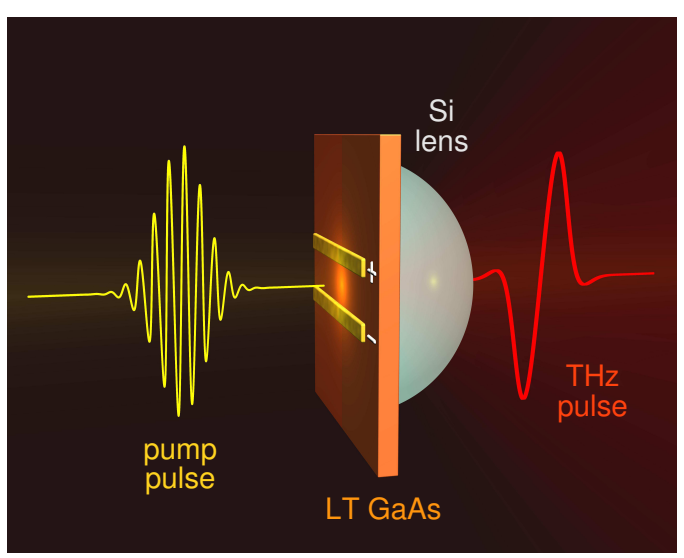

(b)

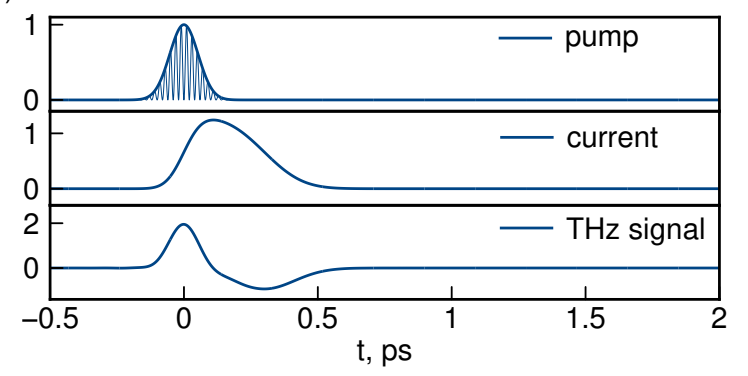

Figure 2 (a) Schematic view of a setup used to generate pulsed $\mathrm{THz}$ radiation by photoconductive LT-GaAs based antenna and (b) time evolution of pump radiation envelope, antenna photocurrent and generated $\mathrm{THz}$ signal.

\subsection{Carrier dynamics in semiconductors under pulsed optical excitation}

Currently, there are many different approaches to $\mathrm{THz}$ generation, in a vast variety of layouts and involving various materials. However, for practical applications, the method involving the excitation of non-equilibrium charge carriers in the surface layer of semiconductor structures by ultrashort optical pulses is the most widely used. Figure 2(a) shows the simplest layout for submillimetre waves generation in semiconductor (LT-GaAs) based photoconductive antennas. Fs-laser (usually Ti:Sapphire [6] or much cheaper fiber-based laser systems such as second [6] or fundamental $[86,89]$ harmonic of Er-doped fiber lasers) serves as a source of ultrashort light pulses with a duration of several tens or hundreds of femtoseconds. The shape of the optical pulse envelope is very important as it induces the nonlinear polarization of the same form (optical rectification), which is capable of increasing the output power of $\mathrm{THz}$ radiation [104, 105]. Silicon lens, shown in Figure 2(a), serves to collect and collimate the generated $\mathrm{THz}$ radiation $[130,131]$ and at the same time as a heatsink to transfer heat excess away from the substrate. Moreover, it works as an anti-reflective element, since the refractive index of $\mathrm{Si}$ in $\mathrm{THz}$ range is equal to $n_{\mathrm{Si}}=3.4$ [132], which is about the same as one of high-resistive GaAs $\left(n_{\mathrm{GaAs}} \approx 3.6\right)$ [132]. 
The dependence of the output power of $\mathrm{THz}$ generation in a semiconductor $P_{\mathrm{THz}}$ on the frequency $\omega_{\mathrm{THz}}$ is given by [133]:

$$
P_{\mathrm{THz}}\left(\omega_{\mathrm{THz}}\right) \backsim \frac{1}{1+\left(\omega_{\mathrm{THz}} \tau_{\mathrm{eff}}\right)^{2}},
$$

where $\tau_{\text {eff }}$ is the effective lifetime of photocarriers, equal to the lifetime of nonequilibrium charge carriers in the semiconductor. From the Eq.(1), it is clearly seen that the shorter the lifetime $\tau_{\text {eff }}$ is, the greater power $P_{\mathrm{THz}}$ can be obtained. Gallium arsenide grown by molecular beam epitaxy on a low-temperature substrate (LT-GaAs) is usually chosen as a photoconductive material, as this growth method allows obtaining a material with a larger number of defects and hence with shorter lifetimes of nonequilibrium charge carriers ( $\leq 200 \mathrm{fs}[133])$. When irradiated by fs-laser pulses with photon energies greater than the bandgap, the concentration of nonequilibrium charge carriers in LT-GaAs rapidly increases, and the electron-hole pairs are formed at the surface layer. In the semiconductor model, all electrons are set to have the same effective mass as they have a low energy because of the wide band of gallium arsenide. This model is not applicable to narrow-gap semiconductors such as indium arsenide (InAs). In the case of InAs, it is necessary to consider ensembles of equilibrium and photoexcited electrons interacting through self-consistent field [134], which greatly complicates the mathematical description of processes.

The localization of charges at surface states in the surface layer, gives rise to the so-called built-in field. The lightinduced electrons and holes are accelerated and separated by this field, and the resulting uneven distribution of charge can be considered as a dipole with the dipole moment equal to the sum of momenta of all induced charges in the volume. The dipole oscillations occur until the equilibrium is established again. During the relaxation, electrons emit energy in the form of electromagnetic waves in the $\mathrm{THz}$ range and the relaxation time determines both spectrum and power of the generated radiation. Built-in surface field plays a very important role in this process. The stronger this field is, the more intense is the emitted THz signal. For this reason, biased metal electrodes are often applied to the semiconductor surface to create a stronger electric field. The mechanism of THz generation enhancement by such structures will be described in detail in the next section of this Review. Since the generation takes place near the air-semiconductor interface, a larger portion of the $\mathrm{THz}$ radiation is emitted into the GaAs substrate, owing to the the greater optical density. The reason for this is the greater optical density of the GaAs in comparison with air. Therefore, highly doped semiconductor materials are not suitable for efficient photoconductive antennas due to their increased absorption in the $\mathrm{THz}$ region of the spectrum [132]. On the other hand, the doping of the sample increases the concentration of photoinduced electron-hole pairs, which, ultimately, increases the current and hence the $\mathrm{THz}$ radiation power. A reasonable compromise between these competing factors (i.e. higher absorption vs. higher current) will substantially improve the efficiency of generation.
Another effective method of increasing the optical-to$\mathrm{THz}$ conversion efficiency is the application of an external magnetic field. It has been shown, that a semiconductor placed in a constant magnetic field of $1 \mathrm{~T}$ generates $\mathrm{THz}$ radiation with efficiency up to 20 times larger than without magnetic field [135]. The optimal direction of magnetic field is the one perpendicular to the semiconductor surface, and $\mathrm{THz}$ pulse amplitude is proportional to the squared amplitude of the magnetic field. This effect is explained by the appearance of the Hall component of the current, excited carriers being driven by external or surface field [134-137]. However, such approach requires the use of expensive magnets that are not suitable for implementation in compact setups.

The analysis of temperature dependence of $\mathrm{THz}$ radiation power shows that with decreasing temperature the power goes up. With the GaAs temperature decreasing from $280 \mathrm{~K}$ down to $80 \mathrm{~K}$, the radiation energy is increased by 3.4 times [138].

\subsection{Pulsed THz generation in photoconductive antennas}

An approach to $\mathrm{THz}$ generation in a semiconductor surface due to the accelerated motion of nonequilibrium charge carriers induced by fs-laser pulses and driven by semiconductor surface field, unfortunately, has low optical-to-terahertz conversion efficiency. To overcome these limits, metal electrodes (usually a nickel-gold-platinum alloy) are deposited onto the substrate surface and are biased with an external voltage to create a high electric field in the gap between them, where the photocarriers are generated, and thus enhancing the generation of the THz waves [139]. Such structures together with a photoconductive semiconductor substrate are referred to as $\mathrm{THz}$ photoconductive antennas. An example and principle of operation of such antenna are shown in Figure 2(a).

The mechanism of $\mathrm{THz}$ generation, in this case, is similar to that discussed in the preceding section. Under an ultrashort laser pulse, electron-hole pairs are formed in the surface layer. The conductivity of the semiconductor as a consequence increases proportionally to the electrons and holes concentration: $\sigma=q n_{\mathrm{e}} \mu_{\mathrm{e}}+q n_{\mathrm{p}} \mu_{\mathrm{p}}$, where $q$ is the elementary charge, $n_{\mathrm{e}}\left(n_{\mathrm{p}}\right)$ is the concentration of electrons (holes), and $\mu_{\mathrm{e}}\left(\mu_{\mathrm{p}}\right)$ is their mobility. The increase of conductivity results in a giant current pulse of photoinduced charges, electrons and holes, being accelerated in opposite directions by the external electric field in the gap. The acceleration of charge carriers by the external field $E_{\text {bias }}$, in this case, can be described by the differential equation:

$$
\frac{d v_{\mathrm{e}, \mathrm{p}}}{d t}=-\frac{v_{\mathrm{e}, \mathrm{p}}}{\tau_{\mathrm{eff}}}+\frac{q E}{m_{\mathrm{e}, \mathrm{p}}},
$$

where $v_{\mathrm{e}, \mathrm{p}}$ is the drift velocity of electrons and holes, $m_{\mathrm{e}, \mathrm{p}}$ are their masses, $\tau_{\text {eff }}$ is approximately $30 \mathrm{fs}$ in lowtemperature GaAs, $E$ is the local field. The latter is related to 
(a)

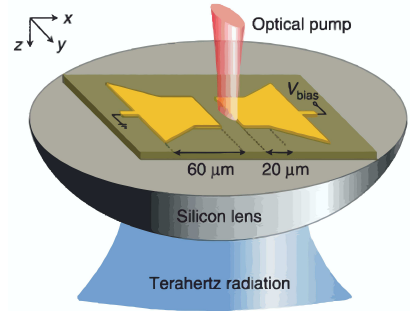

(b)

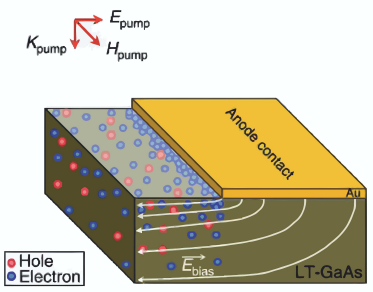

Figure 3 (a) A photoconductive antenna consisting of a GaAs substrate and electrodes arranged in the bow-tie geometry. (b) The distribution of photoinduced electrons near the anode when the bias voltage is applied to the electrodes. [70]

$E_{\text {bias }}$ by $E=E_{\text {bias }}-\frac{P}{\alpha \varepsilon_{0}}$, where $\alpha$ is the static dielectric susceptibility of semiconductor (equal to 3 for the LT-GaAs), $\varepsilon_{0}$ is the vacuum permittivity, and $P$ is the polarization caused by the separation of electrons and holes, which gives the bias threshold of the LT-GaAs of $300 \mathrm{kV} / \mathrm{cm}$. The time evolution of the polarization can be described by the following inhomogeneous differential equation:

$$
\frac{d P}{d t}+\frac{P}{\tau_{\text {rec }}}=J(t)
$$

where $\tau_{\text {rec }}$ is the recombination time of holes and electrons, and $J$ is the density of currents on the surface of the semiconductor defined by: $J(t)=q n_{\mathrm{e}} v_{\mathrm{e}}+q n_{\mathrm{p}} v_{\mathrm{p}}$. From Eqs. (2-3), using Maxwell's equations, one can derive a formula for the intensity of the radiated $\mathrm{THz}$ field at a much larger than THz wavelength $\lambda$ distance $z$ from the source [140]:

$$
E(z, t)=-\frac{A}{4 \pi \varepsilon_{0} c^{2} z} \cdot \frac{d J}{d t}=-\frac{A}{4 \pi \varepsilon_{0} c^{2} z} \cdot\left(q v \frac{d n}{d t}+q n \frac{d v}{d t}\right),
$$

where $n$ is the total concentration of carriers, $v$ is their velocity, $A$ is the area of the irradiated surface (equal to the area of the gap between the electrodes), and $c$ is the speed of light in a vacuum. Thus, the external field increases the energy of charge carriers, and hence the output power of $\mathrm{THz}$ radiation, not influencing, however, the spectral characteristics, as they are determined by the lifetime of electrons and holes. Pump pulse envelope, photocurrent in the surface layer, and resulting $\mathrm{THz}$ field examples obtained with this model are shown in Figure 2(b).

Authors of Refs. [70, 141] have observed that various pump of the semiconductor in the $\mathrm{THz}$ photoconductive antenna gap can affect the output power. Moreover, there are two options for photoconductor pump: uniform illumination of the antenna gap, and partial asymmetric illumination close to the anode (Figure 3(a)). In the case of uniform illumination, the current of photoinduced charge carriers is proportional to the optical power of the pump. However, in the case of asymmetric illumination, the photocurrent and hence the $\mathrm{THz}$ signal are increasing nonlinearly with the optical pump power. This pumping regime is characterized by the increased electric field near the anode, and, conse- (a)

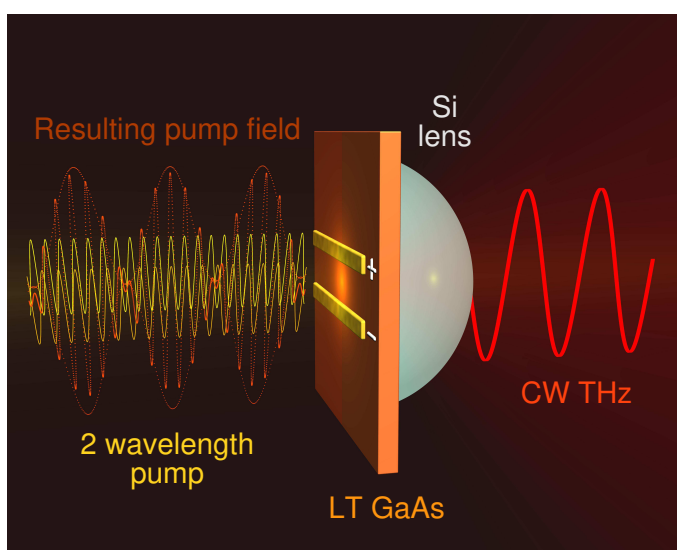

(b)

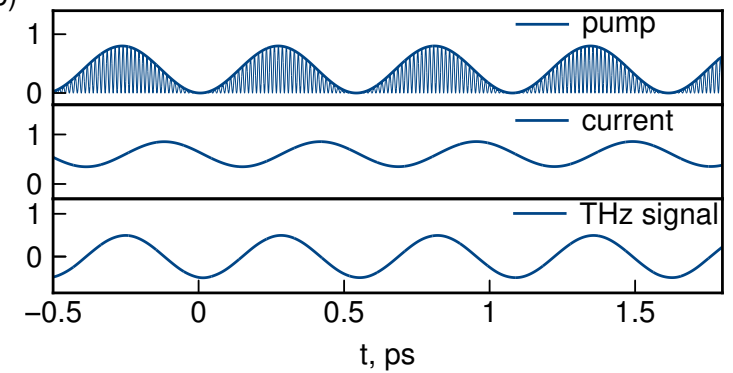

Figure 4 (a) Schematic illustration of CW THz generation by a LTGaAs based photomixer and (b) time evolution of pump radiation envelope, antenna photocurrent and generated $\mathrm{THz}$ signal.

quently, increased electrons concentration in this region (Figure 3(b)). This leads to an increase of the photocurrent, resulting in an enhanced power of the $\mathrm{THz}$ signal. It has been reported that the asymmetric illumination of the photoconductive antenna gap gives more than 4-fold enhancement compared to the uniform illumination [141]. Moreover, it has been shown that the stronger optical field is concentrated in the area of the anode, the more powerful $\mathrm{THz}$ radiation is [142].

\subsection{Continuous $\mathrm{THz}$ generation in photomixers}

The process of $\mathrm{THz}$ heterodyne generation in semiconductors is similar to that discussed in the preceding sections, the only difference being the pump laser regime. Under continuous double-wavelength pump with a frequency difference lying in the $\mathrm{THz}$ region, electron-hole pairs are formed in the surface layer and the conductivity of the semiconductor is modulated proportionally to the electrons and holes concentration. Such devices for photoconductive $\mathrm{CW} \mathrm{THz}$ generation are usually called photomixers [143, 144]. The schematic layout of $\mathrm{CW} \mathrm{THz} \mathrm{generation} \mathrm{in} \mathrm{a} \mathrm{photomixer}$ and its principle of operation are shown in Figure 4(a).

The surface conductivity and charge carriers acceleration in a photomixer are similar to those in a photoconductive antenna, and the number of carrier pairs similarly 
(a)

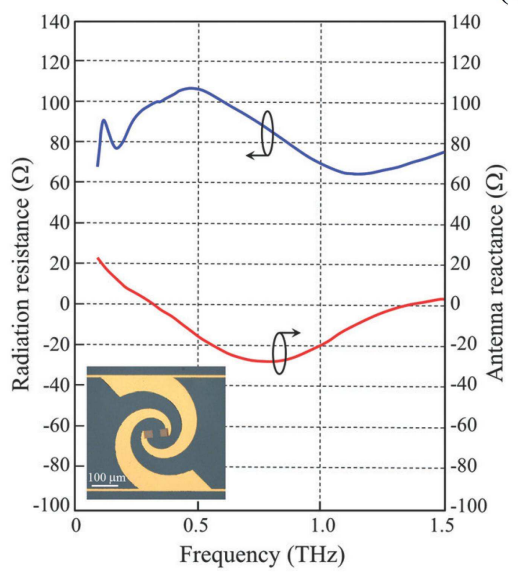

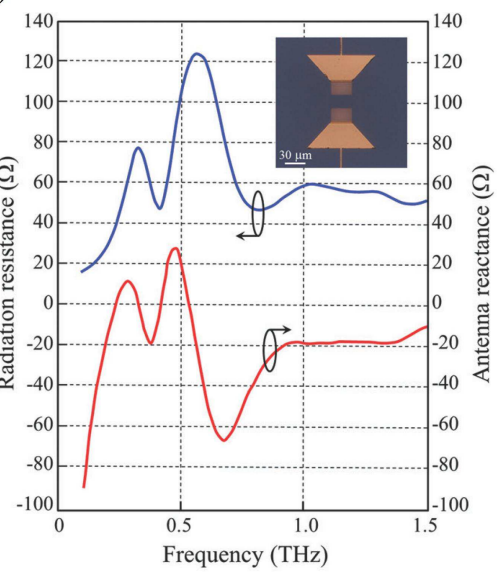

(b)

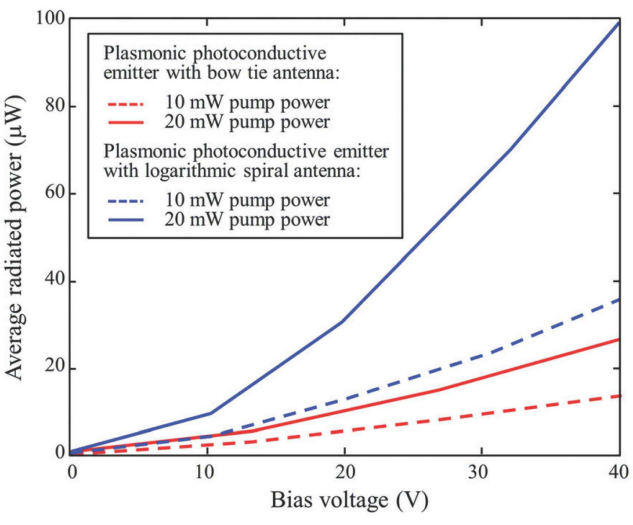

Figure 5 (a) The frequency dependence of impedance (blue) and reactance (red) of two different THz antennas, spiral and bow-tie, respectively. (b) Dependencies of the radiated power of the spiral (blue) and bow-tie (red) antennas on the bias voltage between electrodes for different powers of fs- pump. [88]

follows the pump evolution:

$$
\frac{d n(t)}{d t}=\frac{\eta I_{\mathrm{pump}}(t)}{h v}-\frac{n}{\tau_{\mathrm{rec}}}
$$

where $\eta$ is the external quantum efficiency, $I_{\text {pump }}(t)$ is the pump intensity, $\tau_{\text {rec }}$ is the carrier recombination time, and $h v$ is the energy of the pump photon.

The pump radiation in the case of photomixing comprises two laser beams bringing to the surface average intensities $I_{1}$ and $I_{2}$ at the frequencies $\omega_{1}$ and $\omega_{2}$, respectively. Thus, the intensity at the surface can be described as:

$$
\begin{aligned}
I_{\text {pump }}(t) & =I_{1}+I_{2}+2 \sqrt{I_{1} I_{2}}\left(\cos \left(\omega_{1}-\omega_{2}\right) t+\right. \\
& \left.+\cos \left(\omega_{1}+\omega_{2}\right) t\right) .
\end{aligned}
$$

The second oscillating term in the sum changes within a timescale that is significantly shorter than the carrier effective recombination time $\tau_{\mathrm{c}}$, hence not affecting the photocurrent. Neglecting this term, after substitution of Eq. (6) into Eq. (5), one obtains the evolution of carrier density [143]:

$$
n(t)=\frac{\eta \tau_{\mathrm{rec}}}{h v}\left(I_{1}+I_{2}+\frac{\sqrt{I_{1} I_{2}} \sin (\omega t+\phi)}{\sqrt{1+\omega^{2} \tau_{\mathrm{rec}}^{2}}}\right),
$$

where $\omega=\left(\omega_{1}-\omega_{2}\right)$ is the $\mathrm{THz}$ frequency, and $\phi=$ $\tan ^{-1}\left(\frac{1}{\omega \tau_{\text {rec }}}\right)$ is the phase delay due to the carriers lifetime.

Since the applied field is constant, for the photocurrent we obtain [145]

$$
J(t)=I_{D}+\frac{I_{A}}{\sqrt{1+\omega^{2} \tau_{\mathrm{rec}}^{2}}} \sin (\omega t+\phi),
$$

where $I_{D}=E_{\text {bias }} \mu_{e} \tau_{\text {rec }}\left(I_{1}+I_{2}\right)$ is the DC photocurrent and $I_{A}=2 E_{\text {bias }} \mu_{e} \tau_{\text {rec }} \sqrt{I_{1} I_{2}}$ is the AC component of the current, which is responsible for the generation of the $\mathrm{CW} \mathrm{THz}$ signal:

$$
E_{\mathrm{THz}}(t) \propto \frac{d J(t)}{d t}=\frac{\omega I_{A}}{\sqrt{1+\omega^{2} \tau_{\mathrm{rec}}^{2}}} \cos (\omega t+\phi) .
$$

It can be seen, that for the maximum conversion efficiency, the powers of both wavelength modes should be as close to each other as possible. The pump intensity profile, the corresponding photocurrent, and far field $\mathrm{THz}$ signal of the photomixer calculated with the described model are shown in Figure 4(b).

\subsection{Effect of the contact electrodes shape on the radiative characteristics of $\mathrm{THz}$ photoconductive antennas and photomixers}

One of the most important characteristics of all antennas, including radio, microwave, and $\mathrm{THz}$ photoconductive ones, is the amount of emitted power. However, between the radio or microwave antenna and $\mathrm{THz}$ photoconductive one, there is a significant difference - the nature of the input signal. In the case of a $\mathrm{THz}$ antenna, it is the laser radiation that generates the nonequilibrium electrons and holes in the surface layer of the photoconductor. Therefore, the $\mathrm{THz}$ photoconductive antenna is usually considered as a system consisting of two elements: the photoconductor and the antenna. These elements, in turn, are described by the traditional physical quantities, such as active and reactive conductivity, capacitance, and input impedance [133,141].

The output power $P_{\text {rad }}$ of THz photoconductive antenna can be expressed in terms of antenna resistance and reactance [141]:

$$
P_{\text {rad }}(\omega)=\frac{1}{2}\left|S_{\mathrm{p}}(\omega) P_{\mathrm{opt}}(\omega)\right|^{2} \xi \frac{G_{\mathrm{A}}}{\left(G_{\mathrm{A}}+G_{\mathrm{p}}\right)^{2}+\left(\omega C+B_{\mathrm{A}}\right)^{2}},
$$


where $S_{\mathrm{p}}$ is the current susceptibility of the photoconductor, $P_{\mathrm{opt}}(\omega)$ is the optical pump power at frequency $\omega, \xi$ is the antenna efficiency (which describes the dissipative losses in the antenna material [146]), $G_{\mathrm{A}}$ and $B_{\mathrm{A}}$ are respectively the active and reactive conductances of the antenna, $G_{\mathrm{p}}$ is the conductivity of the photoconductor, and $C$ is its capacitance. Regardless of the $\mathrm{THz}$ photoconductive antenna geometry, in order to maximise its radiated power, its stray conductance must be much less than its active conductance $\left(\omega C+B_{\mathrm{A}}\right) \ll\left(G_{\mathrm{A}}+G_{\mathrm{p}}\right)$. Full or partial compensation of reactive conductance can be achieved by maintaining the inductive load of the antenna at the same level as the capacitive load of the photoconductor, or by adding a negative reactive conductance shunt into the antenna $[147,148]$.

In a number of works $[86,87,149-151]$ it was observed that the geometry of electrodes affects both the spectrum width and the frequency of the maximum $\mathrm{THz}$ radiation intensity. Usually, the electrodes of $\mathrm{THz}$ antennas have the geometry of dipole, logarithmic, spiral, and bow-tie antennas [152-155]. The consideration of the real antenna geometry can be done numerically or experimentally. In Ref. [88] a comparative analysis of two common $\mathrm{THz}$ antenna geometries, spiral and bow-tie, is performed. Figure 5 shows the dependence of radiation resistance and reactance of these antennas on the frequency (a) and the dependence of the radiated power on the bias applied to electrodes for $\mathrm{THz}$ frequency corresponding to the maximum output power (b). It can be seen that the spiral antenna, having a larger radiation resistance and a smaller reactance, emits more effectively than the bow-tie antenna within a wider frequency range. Thus, the maximum active resistance and the minimum absolute value of the reactive resistance are prerequisite for efficient $\mathrm{THz}$ radiation generation and can be adjusted by choosing of the antenna geometry $[153,156]$.

Since active and reactive resistances affect the power emitted by a $\mathrm{THz}$ antenna, the spectral characteristics of the antenna impedance and reactance determine the spectral characteristics of the $\mathrm{THz}$ signal. It has been observed that the antenna with radiation resistance slightly depending on frequency in the $\mathrm{THz}$ range has a wider frequency range of an effective operation $[88,157]$. The spiral and bow-tie geometries discussed above are examples of such antennas. Figure 6 shows the spectrum of $\mathrm{THz}$ signal from the spiral photomixer antenna (white squares) measured by a bolometer. At frequencies between 0.1 and $0.6 \mathrm{THz}$ the signal power is almost constant [133]. On the other hand, antennas with a pronounced resonant impedance, such as full-wavelength dipole antennas (black squares), have narrow-band emission spectrum $[147,158,159]$.

In Ref. [151] the bow-tie THz antennas made in the form of Sierpinski triangles are studied. The results are shown in Figure 7. Due to the presence of subwavelength triangle holes on the antenna surface, the strong plasmon resonances at the frequencies of the antenna radiation are excited. Owing to the special distribution of surface plasmon currents, these $\mathrm{THz}$ fractal antennas have a higher performance than the standard bow-tie antenna. The increasing of fractal degree leads to the enhancement of the number of

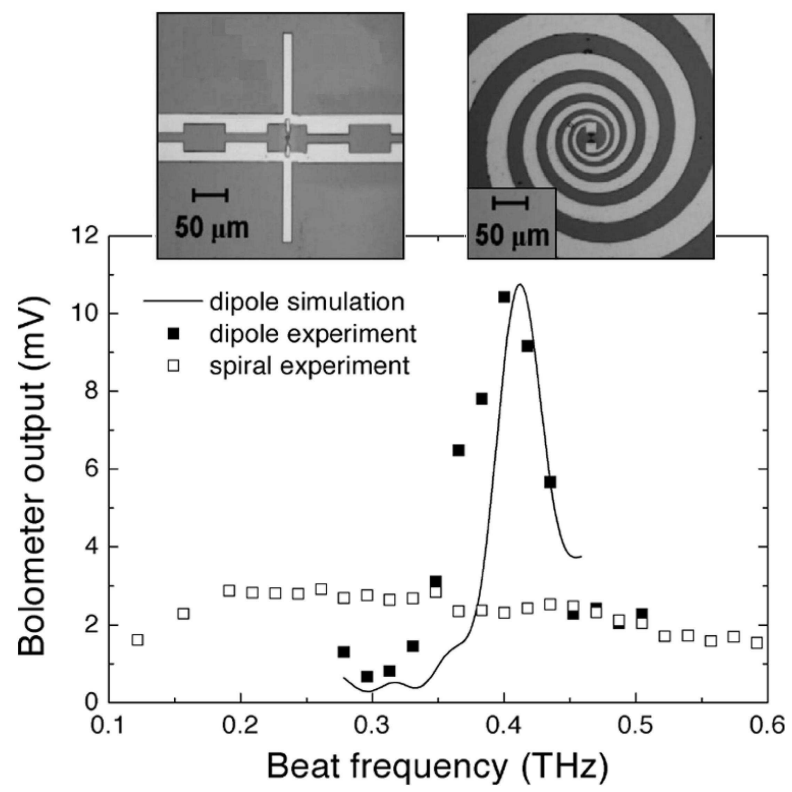

Figure 6 The frequency dependence of the output bolometer signal for the spiral and full-wavelength dipole $\mathrm{THz}$ photomixers. Dots represent experimental data, solid lines - numerical calculations. [133]

plasmon resonances and ultimately to the enhancement of the radiated power.

\section{Hybrid photoconductive THz antennas}

\subsection{Advantages of optical nanoantennas for $\mathrm{THz}$ antennas}

Optical NAs possess all the inherent characteristics of their radio and THz counterparts, except of course that they operate with fields in the optical spectral range. The dimensions of NAs are typically of the order of several hundreds of nanometers. Today, the most widely used and well-studied NAs are the plasmonic ones. They are applied extensively in devices that use photon-electron conversion, such as photodiodes, LEDs, and photovoltaic converters $[77,78,160,161]$. Recently, a number of papers has demonstrated the enhancement of the optical-to-terahertz conversion efficiency in $\mathrm{THz}$ photoconductive antennas with plasmonic NAs. Here we consider the benefits of the optical NAs from the perspective of application for hybrid $\mathrm{THz}$ antennas.

To understand the advantage of optical NAs for THz photoconductive antennas, let us recall that the radiated $\mathrm{THz}$ field amplitude is proportional to photocurrent density [see Eqs.(4) and (9)]. The photocurrent depends on the density of carriers that is a function of time, carriers mobility, and the bias field. It is needed to maximize the photocurrent density to enhance the $\mathrm{THz}$ field generated by the photoconductive antenna. However, the bias field and charge density are 


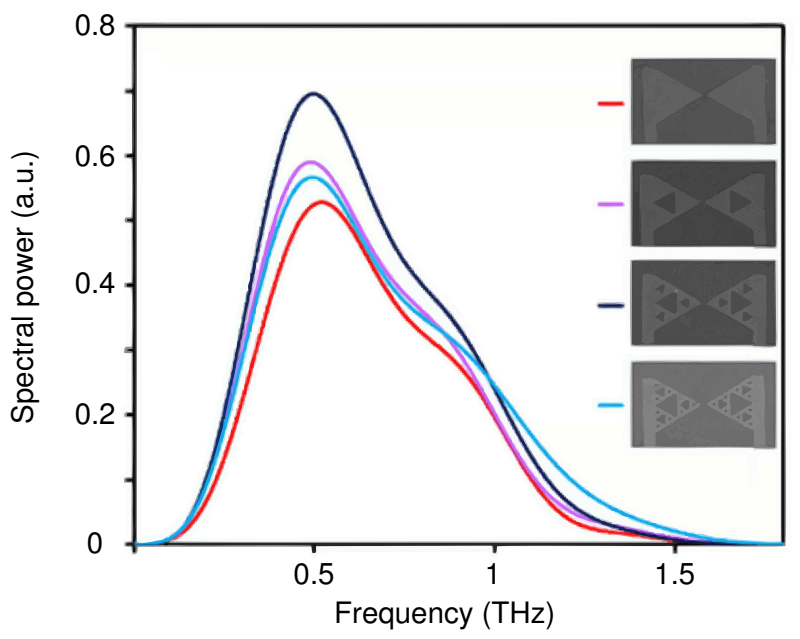

Figure 7 The $\mathrm{THz}$ power spectra for the bow-tie antennas of different fractal degree. [151]

limited by the material breakdown, charge screening, and thermal damage factors. Recently it has been demonstrated that these factors can be considerably improved with the application of optical NAs. The benefits of the optical NAs application including a strong electric field enhancement within semiconductor substrate, reducing the carrier screening effect, and thermal stability enhancement we discuss below.

Strong electric field enhancement. An optical NA, as a part of a hybrid THz-optical photoconductive antenna or photomixer, converts the pump optical radiation into a strong near-field distribution and redistributes the energy within the semiconductor layer. The enhancement of an electric field is described by the local field enhancement factor:

$$
\delta=\frac{|E|}{\left|E_{0}\right|},
$$

which describes by which factor the modulus of the electric field $|E|$ is increased locally (i.e. at a certain point) in the presence of a NA compared to its value $\left|E_{0}\right|$ in the absence of the NA.

The local electric field enhancement occurs due to the plasmon resonance of the NA [69,87, 160-162]. At the same time, the plasmon resonance excited in the metal NA asymmetrically scatters the incident wave, and the field is redistributed in the semiconductor substrate so that the local electric field enhancement factor near the electrodes becomes much greater than the electric field of the incident wave. Therefore, the optical power absorbed by the semiconductor $P_{\mathrm{opt}}$ in the volume $V$ :

$$
P_{\mathrm{opt}}=\frac{1}{2} \int \sigma \delta^{2}\left|E_{0}\right|^{2} d V
$$

increases proportionally to $\delta^{2}$. Here, the value $\sigma$ is a medium conductivity. As a result, the concentration of nonequilibrium charge carriers near the electrodes $n_{\mathrm{e}, \mathrm{p}}$ increases, and, hence the efficiency of optical- to-terahertz
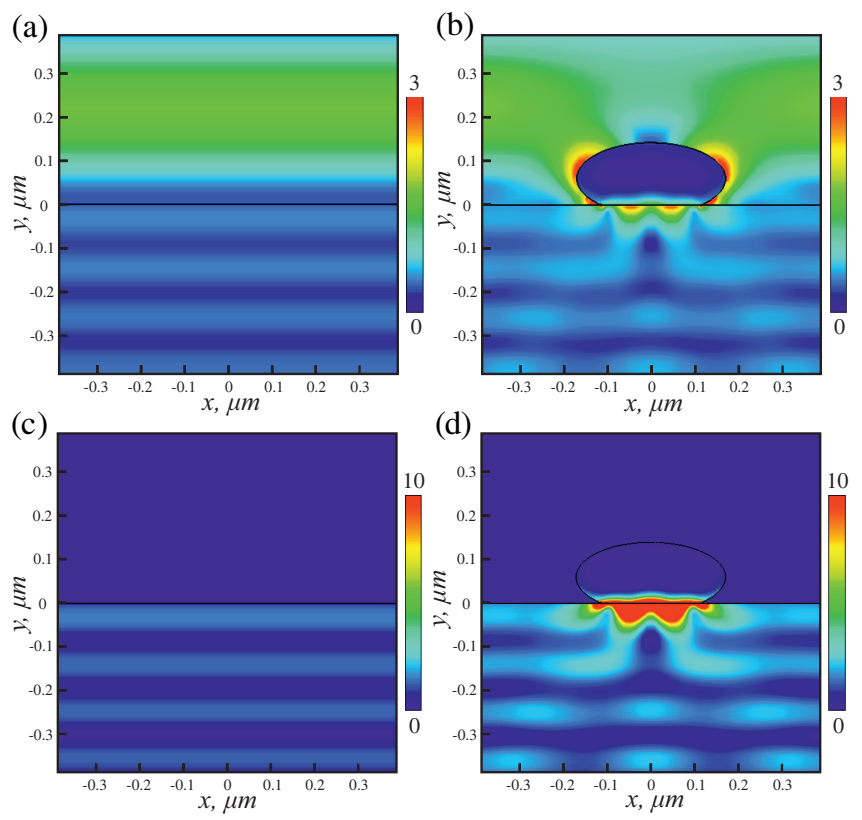

Figure 8 Numerically calculated electric field distribution profiles at the interface between air and the GaAs substrate (a) without and (b) with a silver nanoparticle. Densities of the power absorbed in the GaAs substrate (c) without and (d) with a silver nanoparticle.

conversion increases too, according to Eqs.(4) and (9). The physical dimensions and material of the NAs play an important role in the excitation of the plasmon resonance and the electric field enhancement in the substrate.

As an example, we bring the results of numerical calculations of the electric field distribution at the air-GaAs interface with a Ag spheroid NA and without the NA, which are presented in Figures 8(a,b). The results are obtained by means of the software package CST Microwave Studio. The structure is excited by normally propagated plane electromagnetic wave with the wavelength of $800 \mathrm{~nm}$. The geometrical parameters of the NA correspond to the optimal ones for the excitation of plasmon resonance in such structure at the wavelength of $800 \mathrm{~nm}$. The large semi-axis of the spheroid is $\mathrm{a}=170 \mathrm{~nm}$, minor semi-axis of the spheroid is $b=100 \mathrm{~nm}$. The magnitude of the electric field under the NA (Figure 8(b)) is in about 3 times larger versus the case of the NA absence (Figure 8(a)). Figures 8(c,d) show the distributions of absorbed power densities in the GaAs substrate with and without Ag nanoantenna. It is clearly seen that the power absorbed inside the GaAs in the presence of silver NA is significantly larger (in ten fold) than without the NA.

Reducing of the carrier screening effect. The effectiveness of conventional photoconductive $\mathrm{THz}$ antennas is strongly limited by low drift velocities of the photoinduced charge carriers in semiconductor substrates [71-73]. For an efficient optical-to-terahertz conversion in the frequency range above $0.5 \mathrm{THz}$, the carriers relaxation time $\tau_{\text {rel }}$, as discussed earlier in Section 2 must be subpicosecond. Ac- 
cording to the formula $l=v_{\mathrm{e}, \mathrm{p}} \tau_{\mathrm{rel}}$, the mean free path $l$ is about $100 \mathrm{~nm}$. Therefore, only a small part (about 5\% [70]) of nonequilibrium electrons and holes reaches the electrodes and participate in the efficient $\mathrm{THz}$ generation. The rest of the charge carriers recombines with charges of opposite sign on the way towards the electrodes. This effect is called carrier screening effect $[71,73]$. The diffraction limit for electromagnetic waves does not allow focusing the laser pump into the spot of $100 \mathrm{~nm}$. Therefore, in order to reduce the effect of screening and to improve the properties of photoconductive antennas, optical NAs are used [81].

Thermal stability enhancement. The excess of heat in conventional $\mathrm{THz}$ antennas leads to the thermal generation of carriers after optical pulse excitation [163]. Recombination of thermal and photoinduced carriers in subsequent cycles of charge carriers generation prevents the stable operation of the photoconductive antenna. Recently it has been shown that the optical NAs embedded in a THz photoconductive antenna can improve the thermal stability of the latter due to the large thermal conductivity of metals [74]. For example, gold has the thermal conductivity of $3.14 \mathrm{~W} / \mathrm{cm} \cdot \mathrm{K}$ that is six times larger than the thermal conductivity of GaAs $(0.55 \mathrm{~W} / \mathrm{cm} \cdot \mathrm{K})$. Such higher thermal efficiency also makes the optical NAs very attractive for use in $\mathrm{THz}$ sources.

Thus, the optical NAs being embedded in a THz photoconductive antenna can maximize the transfer of light energy into the absorptive semiconductor substrate and minimize the path length of charge carriers traveling between metallic electrodes. As a result, the optical NAs enhance the performance of the photoconductive $\mathrm{THz}$ antennas. Below, we review the most recent works and organise them according to the approach to optical NA realisation.

\subsection{Plasmon monopole nanoantennas}

The simplest type of NAs applied for hybrid THz antennas is monopole one. The monopole NAs are able to provide a strong electric field enhancement of laser radiation in their vicinity [77]. The optical properties of such NAs dependent on their size, shape and material. Thus, they can be easily tuned to any optical wavelength of operation. The arrays of the monopole NAs can be easily fabricated by means of chemical technics or thermal dewetting [164] even in the gap of $\mathrm{THz}$ photoconductive antennas.

The authors of [165] propose silver plasmonic monopole NAs in the form of so-called nanoislands (Figure 9(a)). The fabrication of such monopole NAs is done by thermal evaporation of silver $(\mathrm{Ag})$, with subsequent deposition onto LT-GaAs antenna substrate surface. This method can be used to pattern nanostructures inside a large area, because of lower costs compared to, for example, electron beam lithography used for the precise fabrication of metal nanostructures [166]. Silver in the hybrid THz-optical antenna is used due to the high quality factor of the silver nanostructures in the visible range, which allows for achieving maximum localization of the field in the semiconductor surface layer with minimal dissipated losses of NAs. In addition, $\mathrm{Ag}$ has a relatively low melting temperature, and
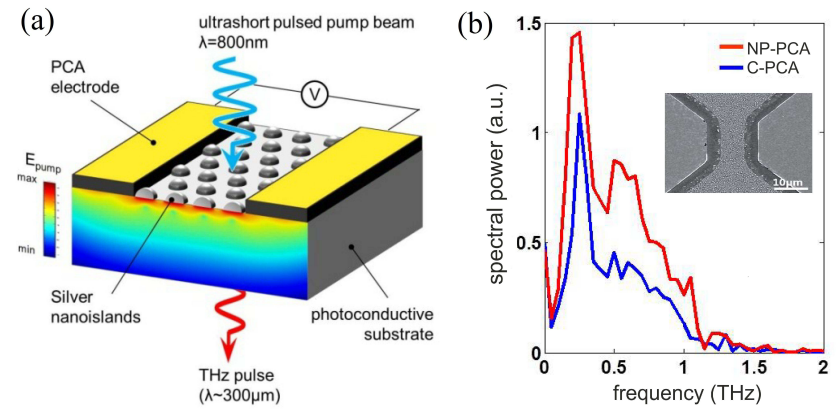

Figure 9 (a) Schematic representation of the photoconductive $\mathrm{THz}$ antenna with a large area of the silver nanoislands (monopole NAs). (b) The power spectra of the $\mathrm{THz}$ radiation for the conventional photoconductive antenna (C-PCA) and hybrid photoconductive antenna (NP-PCA) THz photoconductive antennas. Inset: the SEM image of the silver nanoislands. [165]

therefore its thermal evaporation is possible without thermal damage to the semiconductor GaAs substrate [167]. The geometrical parameters of the monopoles are specially derived so that the plasmon resonances are excited in the structure under an optical pump. For a pump radiation of $800 \mathrm{~nm}$ wavelength, which is typical for Ti:Sapphire fslasers, nanoislands should have a thickness of $20 \mathrm{~nm}$ and a diameter of $173 \mathrm{~nm}$.

Figure 9(b) compares the emission characteristics of a conventional $\mathrm{THz}$ photoconductive antenna and of a hybrid optical- $\mathrm{THz}$ antenna containing nanoislands. It is clearly seen that the hybrid structure has a 1.5-2 times higher conversion efficiency, particularly enhancing the high-frequency part of the generated spectrum. This can be explained by Eq.(4): the monopole NAs concentrate the light within the gaps of adjacent nanoparticles, so that the absorption of optical radiation in these areas increases, growing the concentration of nonequilibrium carriers $n_{e, p}$, which increases the amplitude of the output $\mathrm{THz}$ signal $E_{\mathrm{THz}}$. However, this field redistribution leads to a decrease in its value in the vicinity of nanoislands and significantly reduces the density of electrons and holes in these areas. Nevertheless, this does not affect the total current of photoinduced charges, as silver monopoles are almost perfect conductors for an external electric field. This process is described in detail in Refs. [81, 165].

Another possible realization of hybrid $\mathrm{THz}$ antennas is an array of rectangular monopole NAs arranged inside the photoconductive gap of photoconductive antennas and photomixers [168]. Figure 10(a) schematically shows a hybrid $\mathrm{THz}$ antenna with an array of rectangular nanostructures as well as a SEM image of these nanostructures. The nano-pattern consists of AuGe squares having a diagonal of $500 \mathrm{~nm}$ and a lattice period of $600 \mathrm{~nm}$. This nano-pattern is incorporated in the $\mathrm{THz}$ antenna on the SI-GaAs region between the two electrodes. The mechanism of optical local field enhancement in the vicinity of these NAs is similar to the one in previously described, namely the plasmon resonance excitation. This structure has been realized utilizing 


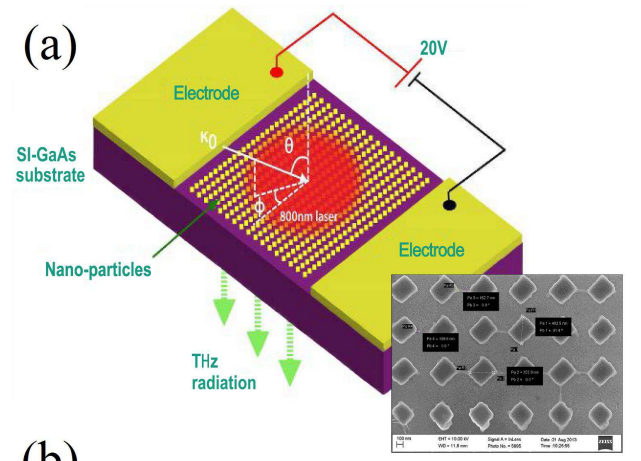

(b)

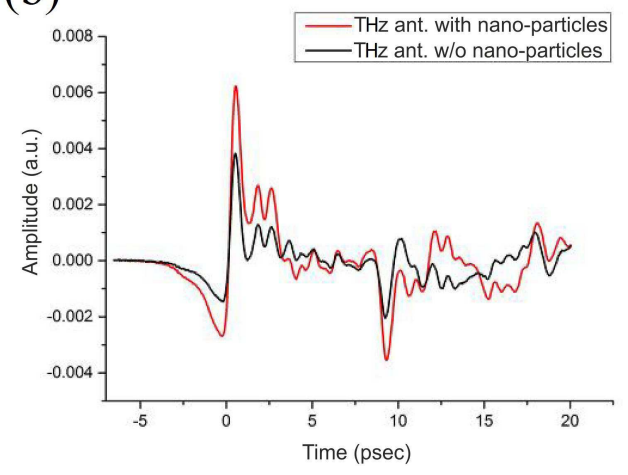

Figure 10 (a) Hybrid THz-optical photoconductive antenna with an array of monopole NAs. The array is fabricated on a substrate of semi-insulating GaAs (SI-GaAs). (b) Output signal of THz photoconductive antennas with (red curve) and without (black curve) monopole nanostructures. [168]

the electron beam lithography. This nanostructured hybrid THz-optical photoconductive antenna is able to double the emitted THz power in comparison with conventional photoconductive antennas (Figure 10(b)).

In Ref. [74], the hybrid $\mathrm{THz}$ antenna with hexagonal metal nanostructures has been proposed and realized. It has been shown that these antennas are superior to plasmonic nanorods-based ones (described below) in THz generation enhancement. Figure 11(a) shows an array of such monopole NAs fabricated inside the photoconductive gap of a dipole $\mathrm{THz}$ antenna using focused ion beam technology. It is known that the increase of pumping intensity increases the photoinduced current, which leads to heating of the semiconductor substrate and eventually to its thermal breakdown $[169,170]$. It has been shown, that an important property of these $\mathrm{THz}$ photoconductive antennas with integrated hexagonal NAs is their high resistance to thermal breakdown [74]. Figure 11(c) demonstrates the advantage of the hexagonal NAs over grating NAs and conventional $\mathrm{THz}$ antennas. To achieve similar photoinduced currents, different antennas require the application of different biases between the electrodes. Moreover, maintaining a predetermined value of the current in the structure with hexagonal monopoles requires less external stress than in other antenna realizations. This suggests that conventional antennas and plasmonic gratings generate more heat than hexagonal NAs, and, accordingly, have a greater risk of experiencing ther- mal breakdown [74]. Figure 11(d) shows the dependence of radiated $\mathrm{THz}$ power on the average power of the fs-laser pump. When comparing the grating and hexagonal NAs, the superior performance of the latter is clearly seen. This is due to a combination of the external field generated by the electrodes, and localization of photoinduced charges in the vicinity to the hexagons tops (Figure 11(b)).

\subsection{Plasmon dipole nanoantennas}

The dipole NAs consist of two monopole ones arranged close to each other [175]. Such structures provide a stronger field localization in the gap between the nanoparticles and larger values of the absorbed power. The metal dipole NAs are used in $\mathrm{THz}$ photoconductive antennas and photomixers, enhancing an optical field of pump laser and redistributing it into the surface layer of the semiconductor substrate near the electrodes. The first implementation of dipole NAs embedded inside the gap of $\mathrm{THz}$ antenna are interdigitated nanostructured electrodes, or as they often called, "nanofingers" [86, 144, 147, 176-178]. Figure 12(a) presents a interdigitated nanostructures embedded in the gap between the electrodes of the logarithmic photomixer. THz photomixers with such nanostructures feature lower parasitic capacitance and, consequently, lower reactance if compared with conventional $\mathrm{THz}$ photomixers, as well as higher optical field localization inside the working area of the antenna (see Figure 12(b)). Hence, photomixers with nanostructured dipole antennas in the photoconductive gap are more effective sources of $\mathrm{CW} \mathrm{THz} \mathrm{radiation} \mathrm{[133]} \mathrm{if} \mathrm{compared}$ to microstructured ones. In some works [171, 172], it has been shown that with increasing the number of dipoles (fingers), the optical-to- $\mathrm{THz}$ conversion efficiency increases (Figure 12(c)). This dependence can be explained by the smaller distance between the dipoles in $\mathrm{THz}$ photomixer with a greater number of fingers, hence increased the degree of the optical field localization in the gap and decreased the effective lifetime of photoinduced charge carriers.

An updated approach to the design of the embedded dipole NAs is the so-called dipole tip-to-tip NAs [173, $174,178]$. Two most commonly used realizations of such nanostructures embedded in a $\mathrm{THz}$ photomixer are shown above. On one side conventional rectangular dipole nanoparticles are shown and trapezoidal bowtie NAs on the other (see Figure 13(a)). Being a continuation of the metal electrodes, these tip-to-tip NAs are designed to reduce the path length of photoinduced electrons and holes [175]. Moreover, due to the nanoscale gap between the dipoles, tipto-tip NAs enhance the localization of the external optical field $[173,178,179]$. High localization of the field in the gap (see Figure 13(b), left picture) occurs because of the surface plasmon excitation due to plasmon resonance at the antenna excitation wavelength [175]. This increases a laser radiation absorption in the semiconductor substrate within the gap, and, consequently, the concentration of charge carriers in the gap. The rapid increase in the charge carriers concentration leads to a huge impulse of the photocurrent that is much greater than in conventional $\mathrm{THz}$ antennas. 
(a)

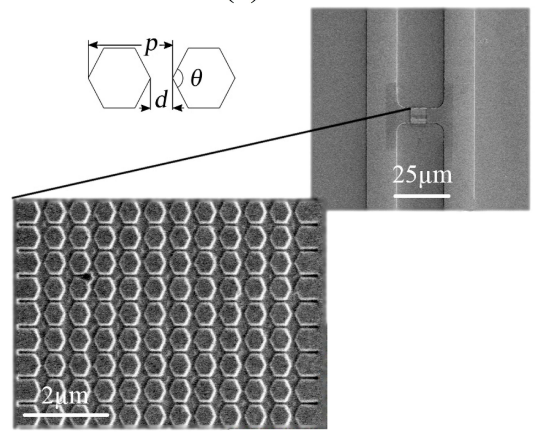

(b)
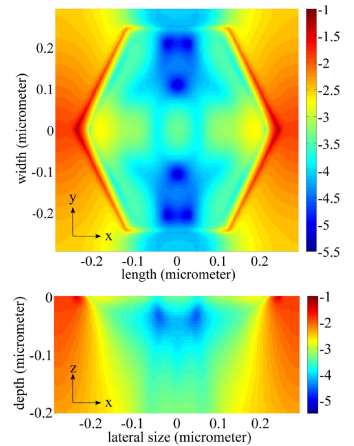

(c)

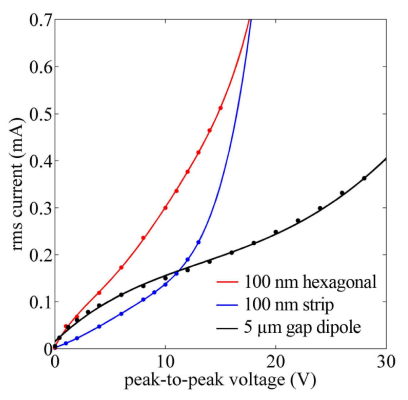

(d)

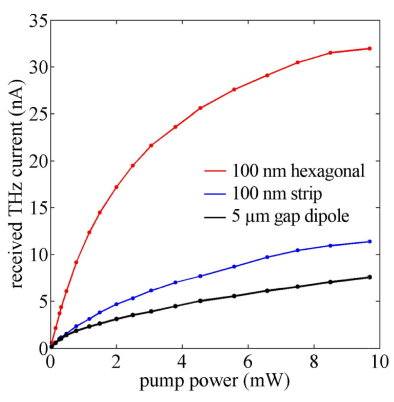

Figure 11 (a) SEM image of periodic array (with period $p$ ) of hexagonal NAs arranged on a SI-GaAs substrate. (b) Electric field distribution profile near the hexagonal NA. (c) Peak-to-peak voltage dependence of photo-excited current in the hybrid THz antenna with hexagonal NAs (red curve), strip plasmonic gratings (blue curve), and the conventional dipole antenna (black curve). (d) Comparison of received $\mathrm{THz}$ currents from the hybrid $\mathrm{THz}$ antennas with hexagonal monopoles, strip plasmonic gratings, and conventional dipole antenna in depends on the pump optical power. [74]

The amplitude of $\mathrm{THz}$ radiation is directly proportional to the rate of change of the photocurrent density, according to Eq.(4), so $\mathrm{THz}$ dipole antenna with integrated optical NAs demonstrate higher output power of THz signal than conventional one at the same pump rate, only because of such nanoscale extensions of the electrodes (Figure 13(c)).

In Ref. [174] it has been noticed, that due to lower capacitive reactive resistance, receiving ability of the bowtie antenna is much better than one of the rectangular-shaped NAs, as a result, the degree of the optical field localization in the trapezoidal NA gap is significantly higher (Figure 13 (c), right picture) [174]. In addition, a smaller capacitance increases the efficiency of generation of $\mathrm{THz}$ radiation by such antenna [133].

\subsection{Two-dimensional plasmonic gratings}

Numerous researchers $[70,81,85-89,150,180-182]$ have shown that on of the most effective implementation of dipole

(a)

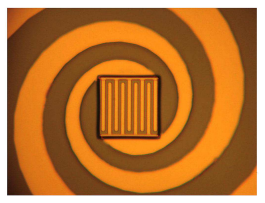

(b)
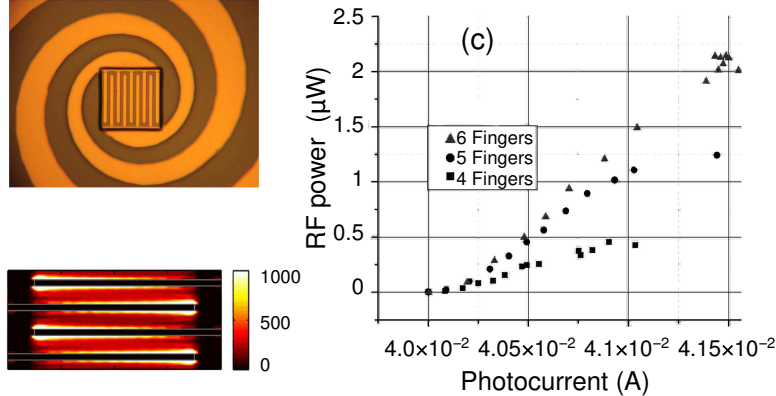

Figure 12 (a) Spiral $\mathrm{THz}$ photomixer with built-in finger NA. (b) Electric field distribution in finger nanostructure of $\mathrm{THz}$ photomixer. (c) Comparison of the radiated power in nanostructured $\mathrm{THz}$ photomixers with a different number of fingers. [171-173]
NAs in the THz photoconductive antennas and photomixers are arrays of metal dipoles whose length is of the order of the gap size between the electrodes. Arrays of dipole plasmonic NAs have received the name of plasmonic gratings or plasmonic nanorod gratings [81].

Figure 14(a) shows the bow-tie $\mathrm{THz}$ photoconductive antenna with embedded plasmonic gratings. Usually, the penetration of light through the apertures is limited by the diffraction limit. However, the geometrical parameters of the gratings can be chosen so that the radiation of a fs-laser with a wavelength of $800 \mathrm{~nm}$ excites the surface plasmon wave in this periodic structure. The excitation of plasmon waves allows the transfer of the most part of the optical pump energy into a semiconductor substrate [183]. The surface plasmon greatly enhances the intensity of the optical field near the metallic electrodes (Figure 14(b)), as a result, the path length of photoinduced electrons and holes to the anode and cathode is reduced in comparison with conventional $\mathrm{THz}$ antennas (Figure 2).

The optical properties of this structure are very sensitive to the polarization of the incident light. When the electric field of the optical pulse is parallel to the plasmonic lattice nanorods, the reflection from the grating surface is sufficiently large. On the contrary, in the case of perpendicular orientation of the electric field vector and nanorods, maximum conversion of incident light into plasmon waves is achieved [81].

The authors of [180] suggest to use unincorporated plasmonic grating in the gap of THz photoconductive switch for enhanced THz pulse generation (Figure 15(a)). Such metal strips of the grating can reduce electrons lifetime in a semiconductor. Antenna and NA were fabricated on a GaAsBi semiconductor substrate characterized with a shorter carrier lifetime than the LT-GaAs substrate [184]. It is shown that when using this structure, more than 2-fold increase of the amplitude of THz signal can be achieved (see Figure 15(b)). However, conversion enhancement in this type of plasmonic 

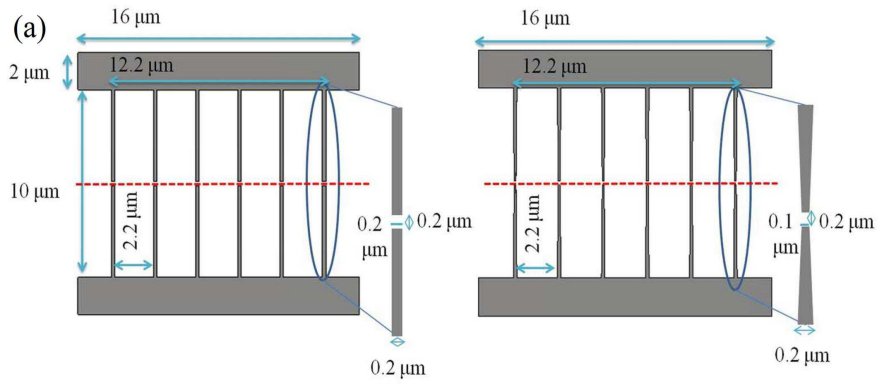

(c)

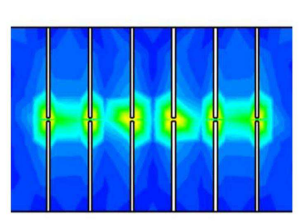

(b)

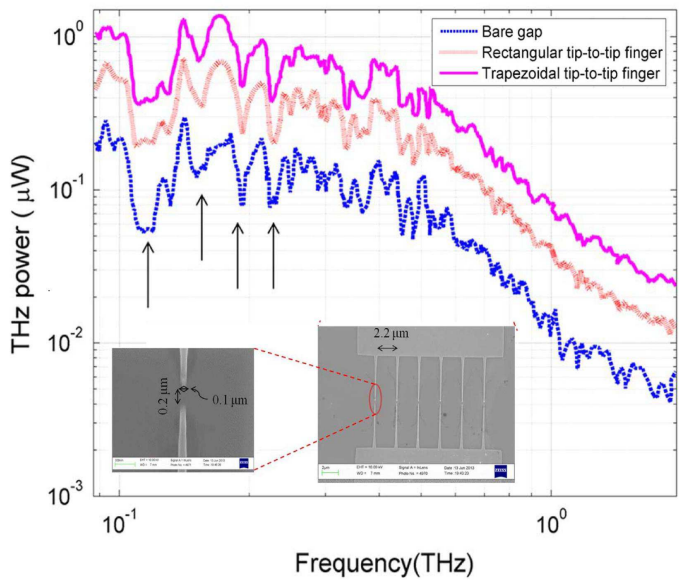

Figure 13 (a) The geometry and (c) electric field distribution profiles of rectangular (left) and trapezoidal (right) dipole NAs embedded in a $\mathrm{THz}$ photoconductive antenna. (b) The spectra of the $\mathrm{THz}$ radiation power for hybrid-optical photoconductive dipole antennas with built-in trapezoidal and rectangular NAs and conventional THz photoconductive antenna. [174]

(a)

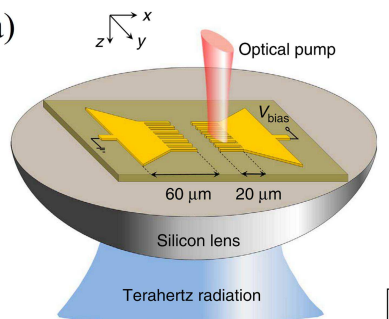

(b)

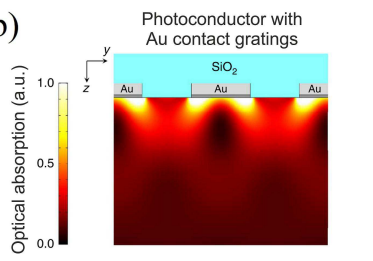

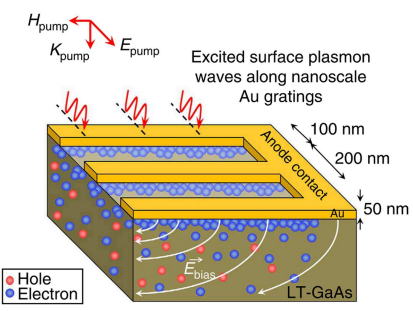

Conventional photoconducto $\Gamma^{x}$

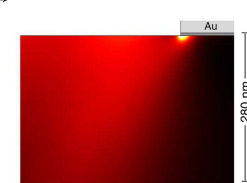

Figure 14 (a) The geometry of the hybrid THz-optical photoconductive antenna and the distribution of electrons and holes near plasmonic nanorods. (b) The image of the distribution of absorbed optical energy in GaAs substrates with plasmonic gratings on the surface and standard $\mathrm{THz}$ photoconductive antenna [70].

grating is low if compared to other methods, due to the large capacitance [180].

Figure 16 depicts another possible implementation of plasmonic nanorod gratings embedded in dipole photoconductive $\mathrm{THz}$ antenna. Instead of comb-like plasmonic grating structures described above, the ends of plasmonic nanorods in this configuration are connected together, which significantly shortens the path of the charge carriers to the electrodes. Moreover, this design allows reducing the capacitive reactance of the $\mathrm{THz}$ antenna that improves its emitting ability and increases the antenna gain coefficient. A disad-

vantage of this plasmonic grating realization is that only half of nonequilibrium electrons and holes reach the electrodes within subpicosecond time. This limits the effectiveness of such structures to $50 \%$ [69].

In order to maintain the operation of the hybrid THzoptical photoconductive antenna without reducing its effectiveness, midway between the anode and the cathode grounding electrode is placed. This electrode is usually embedded into the semiconductor substrate and during a cycle of photoinduced charge carriers generation collects those electrons and holes that reach the electrodes, preventing their slow accumulation in the substrate. In the absence of a grounding electrode, these unwanted carriers recombine
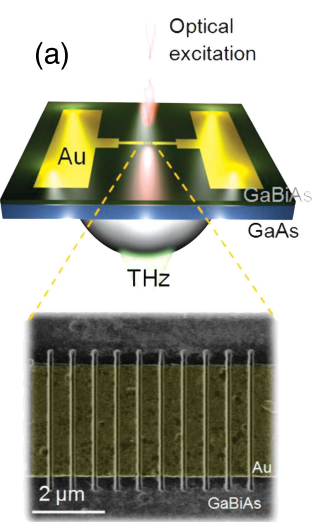

(b)

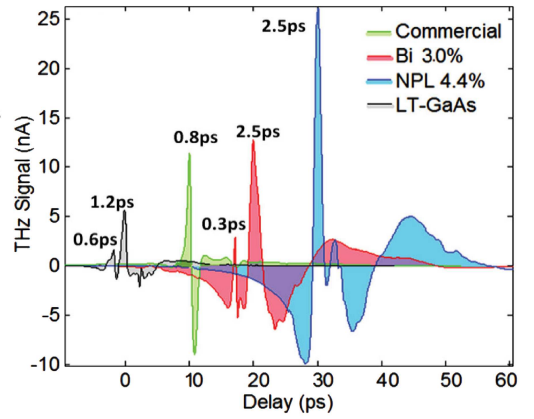

Figure 15 (a) The geometry and SEM image of the THz dipole antenna with the unincorporated plasmonic grating. (b) THz signals of commercial $\mathrm{THz}$ antenna (green curve), dipole antenna with the GaAsBi semiconductor substrate (red curve), dipole antenna with the LT-GaAs substrate (black curve), and hybrid THz-optical antenna with the nanoplasmonic grating (blue curve). 

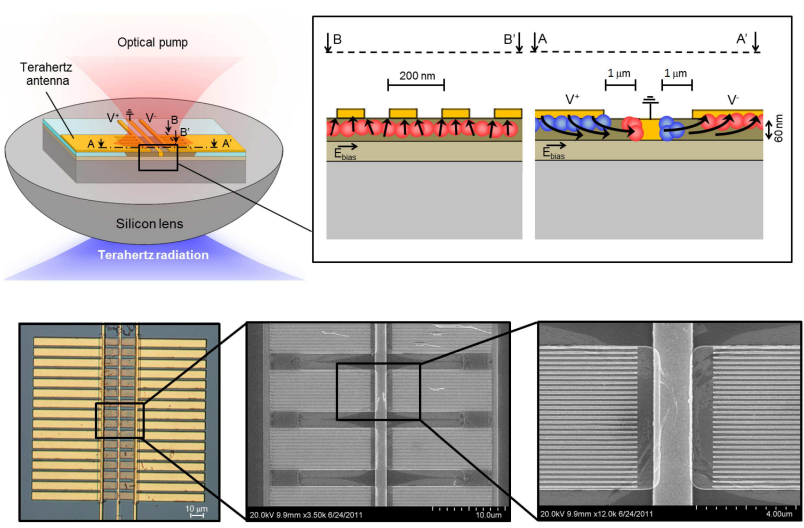

Figure 16 Structural diagram of the $\mathrm{THz}$ photoconductive antenna with embedded plasmonic nanorods and ground electrode, its principle of operation (top). Top view of an array of such antennas and their SEM image (bottom). [69]

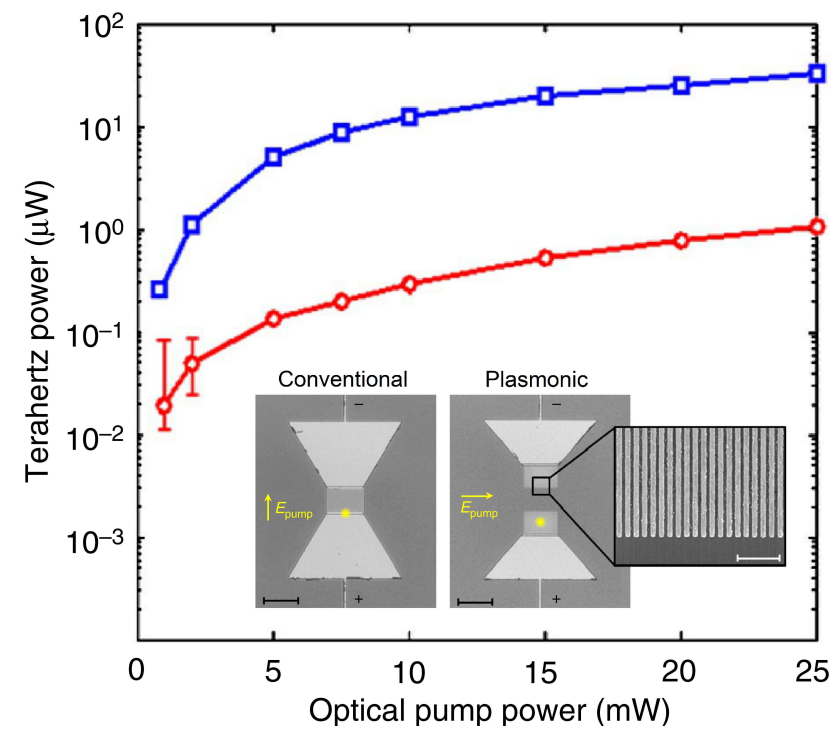

Figure 17 The $\mathrm{THz}$ output power vs. pump power for $\mathrm{THz}$ hybrid optical photoconductive antenna (blue) and a standard $\mathrm{THz}$ photoconductive antenna of the same geometry (red). [70]

with charges of opposite sign of the subsequent cycles of generation, thereby reducing the total number of particles that reach electrodes [69].

To manufacture such structures, the methods of electronbeam lithography, accompanied by the deposition of Au on LT-GaAs substrate are used. A dielectric layer covering NA reduces Fresnel reflection from its surface, thereby increasing the absorption coefficient of the optical pump into the substrate. Usually, $200 \mathrm{~nm}$ thick silica $\left(\mathrm{SiO}_{2}\right)$ is used for these purposes. This coating provides a $70 \%$ absorption of pump light into the semiconductor material.

Comparison of conventional $\mathrm{THz}$ photoconductive antennas and hybrid optical-to- $\mathrm{THz}$ antennas with integrated plasmonic gratings reveals at least one order of magnitude superiority of the latter (Figure 17). Such a significant increase in the output power of $\mathrm{THz}$ radiation is achieved by reducing the path length of charge carriers to the electrodes, thus greatly increasing the photocurrent and decreasing the number of recombined electron-hole pairs.

An important role in THz hybrid optical photoconductive antennas is played by the thickness of the semiconductor substrate, since at a sufficiently large depths (over $100 \mathrm{~nm}$ ) photoinduced charge carriers that do not participate in the effective generation of $\mathrm{THz}$ radiation start to accumulate. These electrons and holes rise from the depths of the substrate to the surface, where they recombine with other charges, thus significantly reducing the ratio of opticalterahertz conversion. To prevent negative effects caused by the non-equilibrium carriers accumulated in the lower layers of the substrate, its thickness should not exceed $100 \mathrm{~nm}$. However, there is a workaround for this problem without reducing the thickness of semiconductor by using threedimensional plasmonic gratings [90].

\subsection{Three-dimensional plasmonic gratings}

Plasmonic gratings described above are two-dimensional nanoscale structures on the semiconductor surface. They provide a high absorption of light quanta by a semiconductor material and significantly increase the concentration of photoinduced charge carriers near metal electrodes. However, the effectiveness of optical-terahertz conversion is still limited by the depth at which charge carriers are generated $(\sim 100 \mathrm{~nm})$. To overcome this limitation, threedimensional plasmonic gratings embedded in the semiconductor substrate are proposed. It has been shown, that such structures allow achieving 7.5\% optical-to- $\mathrm{THz}$ conversion efficiency [90]. Figure 18 (a) shows a schematic of threedimensional plasmonic gratings and distribution pattern of the absorbed optical radiation. Because of its complexity, the manufacturing of such antennas should be discussed in more detail. The process of three-dimensional plasmonic gratings fabrication starts with chemical vapor deposition of a $200 \mathrm{~nm}$ film of $\mathrm{SiO}_{2}$ onto the surface of the semiconductor. Next, on the surface of the $\mathrm{SiO}_{2}$ film, a nanoscale Nickel grid is applied by electron beam lithography. The grid serves as a template layer for etching of $\mathrm{SiO}_{2}$. When using reactive ion etching with a source of inductively coupled plasma a $\mathrm{SiO}_{2}$ grating is formed, which serves as a mask to create nano-walls made of semiconductor material. The plasmonic grating is obtained by deposition of gold on the sidewalls of the resulting semiconductor nanostructures. The height of the walls of the nanostructure determines the order of the modes excited in the resulting subwavelength waveguides. In such a structure waveguide TEM modes of 4th order can be excited by p-component of the fs-laser [185-187]. $\mathrm{A} \mathrm{SiO}_{2}$ mask is then removed by wet etching. Then to reduce the losses associated with reflection, the surface of the antenna is covered with a $200 \mathrm{~nm}$ layer of $\mathrm{SiO}_{2}$. The resulting structure absorbs up to 70 percent of the incident laser radiation [187]. 


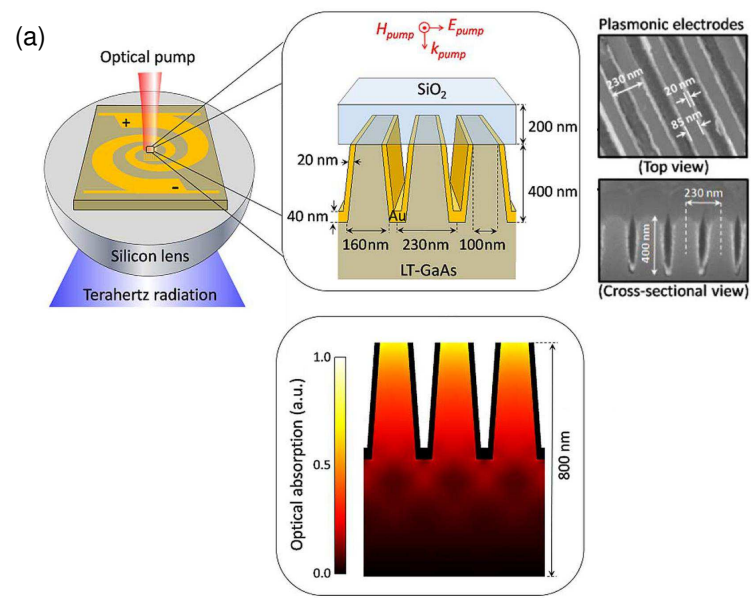

(b)

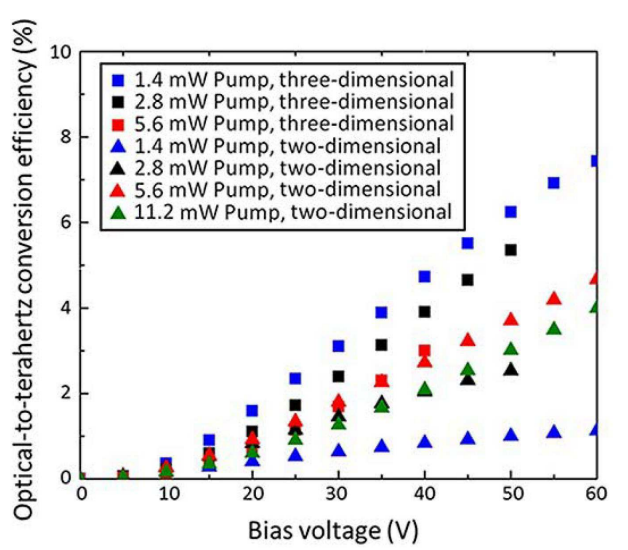

Figure 18 (a) Schematic representation of the logarithmic hybrid THz-optical and photoconductive antenna structures with integrated three-dimensional plasmonic structure, as well as its SEM picture. (b) The dependence of the optical-terahertz conversion on bias applied to antenna electrodes at different pump powers for $\mathrm{THz}$ photoconductive antennas with built-in two-dimensional and three-dimensional plasmonic gratings. [90]

Figure 18(b) demonstrates the advantages of threedimensional gratings two-dimensional It is seen that threedimensional plasmonic grating has a large coefficient of optical-to- $\mathrm{THz}$ conversion in comparison with the twodimensional analogue. At the optical pump power of $1.4 \mathrm{~mW}$, optical-to-THz conversion efficiency reaches $7.5 \%$. Such efficiency increase is due to the fact that the threedimensional plasmonic grating embedded into the surface layer of the semiconductor substrate, hence the contact surface of the grating with the semiconductor is much greater than that of the two-dimensional plasmonic gratings. This increases the concentration of the photoinduced charges near the metal electrodes, which are involved in the effective generation of $\mathrm{THz}$ radiation [90]. However, with increasing optical pump power, optical-to- $\mathrm{THz}$ conversion efficiency in three-dimensional structures decreases. This happens because a large number of separated electron-hole pairs gener- ate a significant electric field with the direction opposite to the external field. The resulting field turns out to decrease, hence the efficiency of $\mathrm{THz}$ generation is reduced [71].

Thus, the $\mathrm{THz}$ photoconductive antennas with integrated three-dimensional plasmonic gratings provide greater efficiency of optical-to- $\mathrm{THz}$ conversion in comparison with two-dimensional plasmonic gratings. In the future, despite the complexity of fabrication technology, these three-dimensional nanoscale structures can replace twodimensional in hybrid THz-optical photoconductive antennas.

\section{Discussion and outlook}

To summarize all results presented in the Review, we have gathered together studies on various approaches to the creation of hybrid photoconductive antennas for $\mathrm{THz}$ generation, in order to facilitate a direct comparison of the proposed methods (see Table 1). We conclude that the most high-performance hybrid $\mathrm{THz}$ photoconductive antennas are the ones based on three-dimensional plasmonic gratings with the maximum conversion efficiency of $7.5 \%$, at the pump power of $1.4 \mathrm{~mW}$. For comparison, hybrid $\mathrm{THz}$ photoconductive antennas based on two-dimensional plasmonic gratings have the maximum conversion efficiency of about $0.2 \%$ only, at a higher pump power $25 \mathrm{~mW}$.

This Review has demonstrated obvious advantages of hybrid $\mathrm{THz}$ photoconductive antennas and photomixers over conventional ones, especially in their conversion efficiency at low pump powers. With the development of compact ultrafast fiber [189] and semiconductor [190,191] lasers, followed by the use of new photoconductive materials suitable for the longer-wavelength, e.g. Yb-based lasers $(1030 \mathrm{~nm})$, Er-doped fiber lasers $(1550 \mathrm{~nm})$ or semiconductor quantum dot lasers (950-1300 nm) pumping, such as GaBiAs [192], InGaAs [193], and also containing ErAs [194], and InAs [66, 129, 195] quantum dots in GaAs substrates, these can lead to the creation of highly effective, miniature $\mathrm{THz}$ transmitters, working at room temperatures both in pulsed and CW regimes. Despite the complexity and high cost of hybrid electrodes fabrication, the total setup cost, that will exclude the extremely expensive titaniumsapphire laser, should significantly decrease.

Moreover, despite a number of plasmonic NAs' advantages associated with their small size and strong electric field localization, such NAs have large dissipative losses resulting in their low efficiency. To overcome such limitations, a new type of nanoantennas based on dielectric nanoparticles (e.g. silicon) with a high index dielectric constant has been recently proposed [196-201]. Such NAs could be very promising for hybrid THz-optical photoconductive antennas. Namely, they also have an ability to strong electric (and magnetic) local field enhancement [202-205]. Moreover, they have a much higher damage threshold due to low dissipative losses. The typical values of damage threshold for metallic nanostructures are: gold nanorods $\left(\sim 70 \mathrm{GW} / \mathrm{cm}^{2}\right.$ or $\sim 10 \mathrm{~mJ} / \mathrm{cm}^{2}$ at 130 fs [206]), gold G-shaped nanostructure $\left(\sim 100 \mathrm{GW} / \mathrm{cm}^{2}\right.$ 


\begin{tabular}{|c|c|c|c|c|c|c|c|}
\hline $\begin{array}{l}\text { Method of Plasmon } \\
\text { Resonance Obtaining }\end{array}$ & Regime & $\begin{array}{l}\text { Fabrication } \\
\text { Complexity }\end{array}$ & $\begin{array}{c}\text { Generation } \\
\text { Enhance- } \\
\text { ment } \\
\text { Factor }\end{array}$ & $\begin{array}{l}\text { Spectral } \\
\text { range, } \\
\mathrm{THz}\end{array}$ & $\begin{array}{l}\text { Maximum } \\
\text { Conversion } \\
\text { Efficiency }\end{array}$ & $\begin{array}{l}\text { At Pump } \\
\text { Power }\end{array}$ & $\begin{array}{c}\# \\
\text { Ref. }\end{array}$ \\
\hline Silver Nanoparticles & Pulse & $\begin{array}{l}\text { Relatively } \\
\text { Low }\end{array}$ & $2 \mathrm{x}$ & $0.1-1.1$ & - & $50 \mathrm{~mW}$ & {$[165]$} \\
\hline AuGe Nanoparticles & Pulse & $\begin{array}{l}\text { Relatively } \\
\text { Low }\end{array}$ & $4 \mathrm{x}$ & $0.1-1.5$ & - & - & [168] \\
\hline Hexagonal Nanoantennas & Pulse & High & $20 \mathrm{x}$ & $0.1-2.4$ & - & $2 \mathrm{~mW}$ & [74] \\
\hline $\begin{array}{l}\text { Interdigitated metallic dipole } \\
\text { nanoantenna (six-nanofinger) }\end{array}$ & $\mathrm{CW}$ & $\begin{array}{l}\text { Relatively } \\
\text { Low }\end{array}$ & - & $0.1-1.1$ & $0.0002 \%$ & $26 \mathrm{~mW}$ & {$[172]$} \\
\hline $\begin{array}{c}\text { Interdigitated metallic dipole } \\
\text { nanoantenna } \\
\text { (five-nanofinger) }\end{array}$ & $\mathrm{CW}$ & $\begin{array}{l}\text { Relatively } \\
\text { Low }\end{array}$ & - & $0.1-1.1$ & $0.0006 \%$ & $50 \mathrm{~mW}$ & [188] \\
\hline $\begin{array}{c}\text { Interdigitated metallic dipole } \\
\text { nanoantenna } \\
\text { (eight-nanofinger) }\end{array}$ & $\mathrm{CW}$ & $\begin{array}{l}\text { Relatively } \\
\text { Low }\end{array}$ & - & $0.1-1.1$ & $0.007 \%$ & $90 \mathrm{~mW}$ & [147] \\
\hline $\begin{array}{c}\text { Rectangular metal dipole } \\
\text { nanoantennas }\end{array}$ & $\mathrm{CW}$ & High & $4 \mathrm{x}$ & $0.1-1.1$ & $0.0005 \%$ & $55 \mathrm{~mW}$ & {$[174]$} \\
\hline $\begin{array}{c}\text { Bow-tie metallic dipole } \\
\text { nanoantennas }\end{array}$ & $\mathrm{CW}$ & High & $7 x$ & $0.1-1.1$ & $0.0008 \%$ & $55 \mathrm{~mW}$ & [174] \\
\hline $\begin{array}{c}\text { Unincorporated plasmonic } \\
\text { gratings }\end{array}$ & Pulse & High & $4 \mathrm{x}$ & $0.1-2.1$ & - & $17 \mathrm{~mW}$ & {$[180]$} \\
\hline $\begin{array}{l}\text { Plasmonic gratings for } \\
\text { impulse } \mathrm{THz} \text { generation }\end{array}$ & Pulse & High & $35 x-50 x$ & $0.1-1.5$ & $0.2 \%$ & $25 \mathrm{~mW}$ & [70] \\
\hline $\begin{array}{l}\text { Plasmonic gratings for } \\
\mathrm{CW}-\mathrm{THz} \text { generation }\end{array}$ & Pulse & High & $20 x$ & $0.2-2.5$ & $0.03 \%$ & $50 \mathrm{~mW}$ & [86] \\
\hline $\begin{array}{l}\text { Plasmonic gratings with } \\
\text { grounding electrode }\end{array}$ & Pulse & High & $50 x$ & $0.1-1.5$ & $0.005 \%$ & $85 \mathrm{~mW}$ & [69] \\
\hline 3D plasmonic gratings & Pulse & $\begin{array}{l}\text { Extremely } \\
\text { High }\end{array}$ & $1500 x$ & $0.1-3.0$ & $7.5 \%$ & $1.4 \mathrm{~mW}$ & [90] \\
\hline
\end{tabular}

Table 1 Comparison of the proposed fabrication methods of hybrid photoconductive antennas for efficient $\mathrm{THz}$ generation.

or $\sim 3 \mathrm{~mJ} / \mathrm{cm}^{2}$ at $30 \mathrm{fs}$ [207]), and gold nanocylinders ( $\sim 200 \mathrm{GW} / \mathrm{cm}^{2}$ or $\sim 20 \mathrm{~mJ} / \mathrm{cm}^{2}$ at 100 fs [208]). According to the known data from literature, low-loss silicon nanoparticles have significantly higher damage threshold: $\sim 400 \mathrm{GW} / \mathrm{cm}^{2}$ or $\sim 100 \mathrm{~mJ} / \mathrm{cm}^{2}$ at 250 fs [209]; and $\sim 1000 \mathrm{GW} / \mathrm{cm}^{2}$ or $\sim 100 \mathrm{~mJ} / \mathrm{cm}^{2}$ at $100 \mathrm{fs}$ [198]. Such considerable difference in damage thresholds for plasmonic (e.g. gold) and all-dielectric (e.g. silicon) materials originates from the difference in their melting temperatures $\left(\mathrm{T}_{m}(\mathrm{Au})=1337 \mathrm{~K}\right.$ and $\left.\mathrm{T}_{m}(\mathrm{Si})=1687 \mathrm{~K}\right)$ and enthalpies of fusion $(\mathrm{H}(\mathrm{Au})=12.5 \mathrm{~kJ} / \mathrm{mol}$ and $\mathrm{H}(\mathrm{Si})=50.2 \mathrm{~kJ} / \mathrm{mol})$. In accordance with these basic parameters, silicon has much higher damage threshold than gold. Therefore, the all-dielectric NAs allow for applying of very high laser intensities which is promising for an effective $\mathrm{THz}$ generation.

Thus, despite a large number of research works in this area and very encouraging results, consensus about the advantage of a particular structure as the absorption enhancer of the pump radiation, and the magnifier of effective generation area in a semiconductor is not formed yet. This is a very young research area where more studies and experiments are needed, aimed primarily at the optimisation of the proposed methods and quest for new types of NAs. In addition, the search for more simple solutions to the problem of transfer of optical radiation into the largest possible nonequilibrium carrier pairs number in a semiconductor, increasing the speed of their movement through the creation of more intense local electric and magnetic fields and reduction of their lifetime by reducing their free path lengths is also needed.

\section{Conclusion}

In this Review, an approach to solving the problem of low energy conversion in $\mathrm{THz}$ photoconductive antennas and photomixers is extensively studied. The reviewed solution comprises the use of optical nanoantennas for enhancing the efficiency of pump laser radiation absorption in the antenna gap, reducing the lifetime of photoexcited carriers and improving antenna thermal efficiency. Within the Review, the general principles of photonic $\mathrm{THz}$ generation in semiconductor converters pumped with fs-optical pulses and double-wavelength $\mathrm{CW}$ laser radiation are described, followed by the particular focus of this work the description of the hybrid THz-optical photoconductive antennas and 
their general principles of operation. Antennas are systematized by the principle of their operation and compared by their efficiency and performance. In particular, the results in this promising field obtained by researchers of hybrid THz-optical photoconductive antennas can be attributed to plasmon monopoles, metal dipole NAs, and 2D/3D plasmon gratings.

\section{Acknowledgments}

This work was supported by the Ministry of Education and Science of the Russian Federation (Projects GZ 2014/190 and GZ No. 3.561.2014/K), and by Russian Foundation for Basic Research (Project 16-07-01166 a). The authors are thankful to Prof. Yuri Kivshar, Dr. Ilya Shadrivov, Dr. Irina Khromova, Mr. Mingzhao Song, and Mr. Ivo Leite for useful discussions.

Key words: terahertz, photoconductive antenna, photomixer, nanoantenna, plasmonic structures

\section{References}

[1] D. G. Martin Van ExterCh Fattinger., Optics Letters 14, 1128-1130 (1989).

[2] B. Ferguson and X.C. Zhang, Nat. Mater. 1(1), 26-33 (2002).

[3] P. H. Siegel, IEEE Transactions on microwave theory and techniques 50, 910-928 (2002).

[4] C. A. Schmuttenmaer, Chem. Rev. 104(4), 1759-1780 (2004).

[5] M. Tonouchi, Nature Photonics 1, 97-105 (2007).

[6] P. U. Jepsen, D. G. Cooke, and M. Koch, Laser \& Photonics Reviews 5(1), 124-166 (2011).

[7] M.F. Pereira, Optical and Quantum Electronics 46(4), 491-493 (2014).

[8] X. T. Xie, B. F. Zhu, and R. B. Liu, New Journal of Physics 15(10), 105015 (2013).

[9] A. W. Wharmby and R. L. Bagley, International Journal of Engineering Science 93, 1-12 (2015).

[10] L. Ozyuzer, A. E. Koshelev, C. Kurter, N. Gopalsami, Q. Li, M. Tachiki, K. Kadowaki, T. Yamamoto, H. Minami, H. Yamaguchi, T. Tachiki, K. E. Gray, W. K. Kwok, and U. Welp, Science 318(5854), 1291-1293 (2007).

[11] U. Welp, K. Kadowaki, and R. Kleiner, Nature Photonics 7(9), 702-710 (2013).

[12] T. Kashiwagi, M. Tsujimoto, T. Yamamoto, H. Minami, K. Yamaki, K. Delfanazari, K. Deguchi, N. Orita, T. Koike, R. Nakayama, T. Kitamura, M. Sawamura, S. Hagino, K. Ishida, K. Ivanovic, H. Asai, M. Tachiki, R. A. Klemm, and K. Kadowaki, Japanese Journal of Applied Physics 51(1R), 010113 (2011).

[13] J. A. Zeitler, P. F. Taday, D. A. Newnham, M. Pepper, K. C. Gordon, and T. Rades, J. Pharm. Pharmacol. 59(2), 209223 (2007).

[14] Y. S. Jin, G. J. Kim, and S. G. Jeon, Journal of the Korean Physical Society 49, 513-517 (2006).
[15] T. Globus, D. Woolard, T. Khromova, T. Crowe, M. Bykhovskaia, B. Gelmont, J. Hesler, and A. Samuels, Journal of Biological Physics 29(2-3), 89-100 (2003).

[16] P. D. Cunningham, N. N. Valdes, F. A. Vallejo, L. M. Hayden, B. Polishak, X. H. Zhou, J. Luo, A. K. Y. Jen, J. C. Williams, and R. J. Twieg, Journal of Applied Physics 109(4), 043505-043505 (2011).

[17] E. P. Parrott and J. A. Zeitler, Applied Spectroscopy 69(1), $1-25$ (2015).

[18] K. Niessen, M. Xu, E. Snell, and A. Markelz, Thz microscopy measurements on inhibitor dependence of protein intramolecular modes, in: Infrared, Millimeter, and Terahertz Waves, (2013).

[19] K. Niessen, M. Xu, E. Snell, V. Cody, J. Pace, M. Schmidt, and A. Markelz, Detection enhancement of protein structural vibrations: Measurements and calculations, in: APS Meeting Abstracts, (2015).

[20] R. J. Falconer and A. G. Markelz, Journal of Infrared, Millimeter, and Terahertz Waves 33(10), 973-988 (2012).

[21] H. Murakami, Y. Toyota, T. Nishi, and S. Nashima, Chemical Physics Letters 519, 105-109 (2012).

[22] J. F. Federici, B. Schulkin, F. Huang, D. Gary, R. Barat, F. Oliveira, and D. Zimdars, Semiconductor Science and Technology 20, S266 (2005).

[23] J. F. Federici, B. Schulkin, F. Huang, D. Gary, R. Barat, F. Oliveira, and D. Zimdars, Semicond. Sci. Technol. 20(7), S266-S280 (2005).

[24] C. Corsi and F. Sizov, THz and Security Applications: Detectors, Sources and Associated Electronics for $\mathrm{THz}$ Applications (Springer Netherlands, 2014).

[25] M. F. Pereira and O. Shulika, Terahertz and Mid Infrared Radiation: Detection of Explosives and CBRN (Using Terahertz) (Springer Netherlands, 2014).

[26] F. Xin, H. Su, and Y. Xiao, Terahertz imaging system for remote sensing and security applications, in: Antennas and Propagation (APCAP), (2014), pp. 1335-1338.

[27] M. Massaouti, C. Daskalaki, A. Gorodetsky, A. D. Koulouklidis, and S. Tzortzakis, Appl. Spectrosc.(11), 1264-1269.

[28] X. Wu, B. Quan, X. Pan, X. Xu, X. Lu, C. Gu, and L. Wang, Biosensors and Bioelectronics 42, 626-631 (2012).

[29] H. R. Park, K. J. Ahn, S. Han, Y. M. Bahk, N. Park, and D. S. Kim, Nano Lett. 13, 1782-1786 (2013).

[30] S. J. Park, J. T. Hong, S. J. Choi, H. S. Kim, W. K. Park, S. T. Han, J. Y. Park, S. Lee, D. S. Kim, and Y. H. Ahn, Scientific Reports 4, 4988 (2014).

[31] L. Xie, W. Gao, J. Shu, Y. Ying, and J. Kono, Scientific Reports 5, 8671 (2015).

[32] M. Zhang and J. T. Yeow, IEEE Nanotechnology Magazine 10, 30-38 (2016).

[33] T. S. Bui, T.D. Dao, L.H. Dang, L. D. Vu, A. Ohi, T. Nabatame, Y. Lee, T. Nagao, and C. V. Hoang, Scientific Reports 6, 32123 (2016).

[34] A. G. Davies, E. H. Linfield, and M. B. Johnston, Phys. Med. Biol. 47(21), 3679-3689 (2002).

[35] P. H. Siegel, Microwave Symposium Digest, 2004 IEEE MTT-S International 3, 1575-1578 (2004).

[36] M. Kawase, T. Saito, M. Ogama, H. Uejima, Y. Hatsuda, S. Kawanishi, Y. Hirotani, M. Myotoku, K. Ikeda, H. Konishi, I. Iga, J. Yamakawa, S. Nishizawa, K. Yamamoto, and M. Tani, Analytical Sciences 27(2), 209 (2011). 
[37] C. Yu, S. Fan, Y. Sun, and E. Pickwell-MacPherson, Quantitative Imaging in Medicine and Surgery 2(1), 33 (2012).

[38] P. C. Ashworth, E. Pickwell-MacPherson, E. Provenzano, S. E. Pinder, A. D. Purushotham, M. Pepper, and V. P. Wallace, Opt. Express 17(15), 12444-12454 (2009).

[39] V. P. Wallace, A. J. Fitzgerald, E. Pickwell, R. J. Pye, P. F. Taday, N. Flanagan, and T. Ha, Appl. Spectrosc. 60(10), 1127-1133 (2006).

[40] K. Shiraga, Y. Ogawa, T. Suzuki, N. Kondo, A. Irisawa, and M. Imamura, J. Infrared, Millimeter, Terahertz Waves 35(5), 493-502 (2014).

[41] L. Rong, T. Latychevskaia, C. Chen, D. Wang, Z. Yu, X. Zhou, Z. Li, H. Huang, Y. Wang, and Z. Zhou, Sci. Rep. 5, 8445 (2015).

[42] K. Coppin, J. Geach, I. Smail, L. Dunne, A. Edge, R. Ivison, S. Maddox, R. Auld, M. Baes, S. Buttiglione et al., Monthly Notices of the Royal Astronomical Society 416(1), 680-688 (2011).

[43] F. Wyrowski, R. Güsten, K. Menten, H. Wiesemeyer, and B. Klein, Astronomy \& Astrophysics 542, L15 (2012).

[44] U. U. Graf, C. E. Honingh, K. Jacobs, and J. Stutzki, Journal of Infrared, Millimeter, and Terahertz Waves 36(10), 896-921 (2015).

[45] A. V. Smirnov, A. M. Baryshev, S. V. Pilipenko, N. V. Myshonkova, V. B. Bulanov, M. Y. Arkhipov, I. S. Vinogradov, S. F. Likhachev, and N. S. Kardashev, Space mission millimetron for terahertz astronomy, in: Space Telescopes and Instrumentation 2012: Optical, Infrared, and Millimeter Wave, (2012).

[46] H. Maezawa, IEICE Transactions on Electronics 98(3), 196-206 (2015).

[47] A. Rogalski and F. Sizov, Opto-Electronics Review 19(3), 346-404 (2011).

[48] I. F. Akyildiz, J. M. Jornet, and C. Han, Phys. Commun. 12, 16-32 (2014).

[49] T. Kürner and S. Priebe, J. Infrared, Millimeter, Terahertz Waves 35(1), 53-62 (2014).

[50] J. Federici and L. Moeller, Journal of Applied Physics 107(11), 111101 (2010).

[51] F. Hu and S. Lucyszyn, Noise analysis for multi-channel thz torchthermal infrared wireless communications systems, in: Microwave Conference (APMC), 2014 AsiaPacific, (2014).

[52] S. Koenig, D. Lopez-Diaz, J. Antes, F. Boes, R. Henneberger, A. Leuther, A. Tessmann, R. Schmogrow, D. Hillerkuss, R. Palmer et al., Nature Photonics 7(12), 977-981 (2013).

[53] C. L. Pan and J. W. Shi, 3 ultrawide-band sub-thz photonic wireless links, in: Microwave Photonics, (CRC Press, 2013), p. 97.

[54] Y. Karisan and K. Sertel, Wideband impedance-matched integrated transceivers for future thz-band wireless networks, in: Radio Science Meeting (USNC-URSI NRSM), 2013 US National Committee of URSI National, (2013), pp. $1-1$.

[55] C. Wang, B. Lu, C. Lin, Q. Chen, L. Miao, X. Deng, and J. Zhang, Terahertz Science and Technology, IEEE Transactions on 4(1), 75-85 (2014).

[56] J. O. Richardson, D. J. Wales, S. C. Althorpe, R.P. McLaughlin, M. R. Viant, O. Shih, and R. J. Saykally, The Journal of Physical Chemistry A 117(32), 6960-6966 (2013).
[57] J. Xu, K. W. Plaxco, and S. J. Allen, Journal of chemical physics 124(3), 36101 (2006).

[58] G. Siles, J. M. Riera, P. Garcia del Pino et al., Antennas and Propagation Magazine, IEEE 57(1), 48-61 (2015).

[59] S. U. Hwu, K. DeSilva, and C. Jih, Terahertz (thz) wireless systems for space applications, in: Sensors Applications Symposium (SAS), 2013 IEEE, (2013), pp. 171-175.

[60] G. Ducournau, P. Szriftgiser, A. Beck, D. Bacquet, F. Pavanello, E. Peytavit, M. Zaknoune, T. Akalin, and J.F. Lampin, Terahertz Science and Technology, IEEE Transactions on 4(3), 328-337 (2014).

[61] A. J. Seeds, H. Shams, M. J. Fice, and C. C. Renaud, Journal of Lightwave Technology 33(3), 579-587 (2015).

[62] K. Vodopyanov, Laser Photonics Rev. 2, 11-25 (2008).

[63] D. Saeedkia and S. Safavi-Naeini, J. Light. Technol. 26(15), 2409-2423 (2008).

[64] T. Otsuji, T. Watanabe, S. Boubanga Tombet, A. Satou et al., Terahertz Science and Technology, IEEE Transactions on 3(1), 63-71 (2013).

[65] I. Wilke, P. S. Dutta, and E. Slobodtchikov, Thz radiation emission from inpas and gainas crystals, in: Infrared, Millimeter, and Terahertz waves (IRMMW-THz), 2014 39th International Conference on, (2014), p. 1.

[66] R. R. Leyman, A. Gorodetsky, N. Bazieva, G. Molis, A. Krotkus, E. Clarke, and E. U. Rafailov, Laser Photon. Rev. 10(5), 772-779 (2016).

[67] M. B. Johnston, Journal of Infrared, Millimeter, and Terahertz Waves 33(4), 391-392 (2012).

[68] M. Tani, K. Yamamoto, E. S. Estacio, C. T. Que, H. Nakajima, M. Hibi, F. Miyamaru, S. Nishizawa, and M. Hangyo, Journal of Infrared, Millimeter, and Terahertz Waves 33(4), 393-404 (2012).

[69] C. W. Berry and M. Jarrahi, New Journal of Physics 14, 105029 (2012)

[70] C. Berry, N. Wang, M. Hashemi, M. Unlu, and M. Jarrahi, Nature Communications 4, 1622 (2013).

[71] G. C. Loata, M. D. Thomson, T. Löffler, and H. G. Roskos, Applied Physics Letters 91(23), 232506 (2007).

[72] T. Hattori, K. Egawa, S.i. Ookuma, and T. Itatani, Japanese journal of applied physics 45(4L), L422 (2006).

[73] Z. Piao, M. Tani, and K. Sakai, Japanese Journal of Applied Physics 39(1R), 96 (2000).

[74] A. Jooshesh, L. Smith, M. Masnadi-Shirazi, V. BahramiYekta, T. Tiedje, T. E. Darcie, and R. Gordon, Optics express 22(23), 27992-28001 (2014).

[75] K. B. Crozier, A. Sundaramurthy, G. S. Kino, and C. F. Quate, J. Appl. Phys. 94, 4632 (2003).

[76] P. Biagioni, J. S. Huang, and B. Hecht, Rep. Prog. Phys. 75, 24402 (2012).

[77] A.E. Krasnok, I. S. Maksymov, A. I. Denisyuk, P. A. Belov, A. E. Miroshnichenko, C. R. Simovski, and Y. S. Kivshar, Physics-Uspekhi 56(6), 539 (2013).

[78] P. Bharadwaj, B. Deutsch, and L. Novotny, Advances in Optics and Photonics 1, 438-483 (2009).

[79] X. Ni, N. K. Emani, A. V. Kildishev, A. Boltasseva, and V. M. Shalaev, Science 335(6067), 427-427 (2012).

[80] A. E. Miroshnichenko, I. S. Maksymov, A. R. Davoyan, C. Simovski, P. Belov, and Y.S. Kivshar, Physica Status Solidi (RRL)-Rapid Research Letters 5(9), 347-349 (2011).

[81] S. G. Park, K. H. Jin, M. Yi, J. C. Ye, J. Ahn, and K. H. Jeong, ACS nano 6(3), 2026-2031 (2012). 
[82] S. K. Patel and C. Argyropoulos, EPJ Appl. Metamat. 2, 4 (2015).

[83] L. Novotny and N. van Hulst, Nature Photonics 5, 83-90 (2011).

[84] A. E. Krasnok, A. P. Slobozhanyuk, C. R. Simovski, S. A. Tretyakov, A. N. Poddubny, A. E. Miroshnichenko, Y.S. Kivshar, and P. A. Belov, Scientific Reports 5, 12956 (2015).

[85] M. Jarrahi, IEEE Trans. Terahertz Sci. Technol. 5, 391397 (2015).

[86] C. W. Berry, M. R. Hashemi, S. Preu, H. Lu, A. C. Gossard, and M. Jarrahi, Optics Letters 39, 4522-4524 (2014).

[87] C. Berry, M. Hashemi, and M. Jarrahi, Journal of Infrared, Millimeter, and Terahertz Waves 33(4), 393-404 (2013).

[88] C. W. Berry, M. R. Hashemi, and M. Jarrahi, Applied Physics Letters 104(8), 081122 (2014).

[89] C. W. Berry, M. R. Hashemi, S. Preu, H. Lu, A. C. Gossard, and M. Jarrahi, Applied Physics Letters 105(1), 011121 (2014).

[90] S. H. Yang, M. R. Hashemi, C. W. Berry, and M. Jarrahi, Terahertz Science and Technology, IEEE Transactions on 4(5), 575-581 (2014).

[91] O. Mitrofanov, I. Brener, T. S. Luk, and J. L. Reno, ACS Photonics 2(12), 1763-1768 (2015).

[92] L. Ju, B. Geng, J. Horng, C. Girit, M. Martin, Z. Hao, H. a. Bechtel, X. Liang, A. Zettl, Y. R. Shen, and F. Wang, Nat. Nanotechnol. 6(10), 630-634 (2011).

[93] M. Shalaby, H. Merbold, M. Peccianti, L. Razzari, G. Sharma, T. Ozaki, R. Morandotti, T. Feurer, A. Weber, L. Heyderman, B. Patterson, and H. Sigg, Appl. Phys. Lett. 99(4), 041110 (2011).

[94] M. Shalaby, J. Fabiańska, M. Peccianti, Y. Ozturk, F. Vidal, H. Sigg, R. Morandotti, and T. Feurer, Appl. Phys. Lett. 104(17), 171115 (2014).

[95] L. Razzari, A. Toma, M. Shalaby, M. Clerici, R. P. Zaccaria, C. Liberale, S. Marras, I. a. I. Al-Naib, G. Das, F. De Angelis, M. Peccianti, A. Falqui, T. Ozaki, R. Morandotti, and E. Di Fabrizio, Opt. Express 19(27), 26088 (2011).

[96] L. Razzari, A. Toma, M. Clerici, M. Shalaby, G. Das, C. Liberale, M. Chirumamilla, R. P. Zaccaria, F. Angelis, M. Peccianti, R. Morandotti, and E. Fabrizio, Plasmonics 8(1), 133-138 (2012).

[97] C. Feuillet-Palma, Y. Todorov, A. Vasanelli, and C. Sirtori, Scientific Reports 3, 1361 (2013).

[98] A. Toma, S. Tuccio, M. Prato, F. De Donato, A. Perucchi, P. Di Pietro, S. Marras, C. Liberale, R. Proietti Zaccaria, F. De Angelis, L. Manna, S. Lupi, E. Di Fabrizio, and L. Razzari, Nano Lett. 15(1), 386-391 (2015).

[99] M. G. Krishna, S. D. Kshirsagar, and S.P. Tewari, Terahertz Emitters, Detectors and Sensors: Current Status and Future Prospects (INTECH Open Access Publisher, 2012).

[100] S. Winnerl, Journal of Infrared, Millimeter, and Terahertz Waves 33(4), 431-454 (2012).

[101] K. Reimann, Reports Prog. Phys. 70(10), 1597-1632 (2007).

[102] V. Apostolopoulos and M. Barnes, Journal of Physics D: Applied Physics 47(37), 374002 (2014).

[103] M.B. Johnston, D. M. Whittaker, A. Corchia, A. G. Davies, and E. H. Linfield, Phys. Rev. B 65, 165301 (2002).

[104] J. A. Fülöp, L. Pálfalvi, M. C. Hoffmann, and J. Hebling, Opt. Express 19, 15090-15097 (2011).
[105] K. Ravi, W. R. Huang, S. Carbajo, E. A. Nanni, D. N. Schimpf, E. P. Ippen, and F. X. Kärtner, Optics express 23(4), 5253-5276 (2015).

[106] E. Abraham, M. Cornet, A. O. Hamouda, J. Degert, and E. Freysz, Terahertz-field-induced second harmonic generation through pockels effect in gallium phosphide crystal, in: CLEO: QELS_Fundamental Science, (2014).

[107] K. Y. Kim, a. J. Taylor, J. H. Glownia, and G. Rodriguez, Nat. Photonics 2(10), 605-609 (2008).

[108] A. Gorodetsky, A. D. Koulouklidis, M. Massaouti, and S. Tzortzakis, Phys. Rev. A - At. Mol. Opt. Phys. 89(3), 1-6 (2014).

[109] P. Maine, D. Strickland, P. Bado, M. Pessot, and G. Mourou, IEEE J. Quantum Electron. 24(2), 398-403 (1988).

[110] M. W. McCutcheon, D. E. Chang, Y. Zhang, M. D. Lukin, and M. Loncar, Opt. Express 17(22), 9 (2009).

[111] C. J. Docherty and M. B. Johnston, J. Infrared, Millimeter, Terahertz Waves 33(8), 797-815 (2012).

[112] L. Luo, I. Chatzakis, J. Wang, F. B. P. Niesler, M. Wegener, T. Koschny, and C. M. Soukoulis, Nat. Commun. 5, 3055 (2014).

[113] D. K. Polyushkin, I. Marton, P. Racz, P. Dombi, E. Hendry, and W. L. Barnes, Phys. Rev. B 89, 125426 (2014).

[114] A. Atrashchenko, A. Arlauskas, R. Adomavicius, A. Korotchenkov, V.P. Ulin, P. Belov, A. Krotkus, and V.P. Evtikhiev, Appl. Phys. Lett. 105, 191905 (2014).

[115] L. Zhang, F. Buccheri, C. Zhang, and X. C. Zhang, Appl. Phys. Lett. 107, 071107 (2015).

[116] T. Seifert, S. Jaiswal, U. Martens, J. Hannegan, L. Braun, P. Maldonado, F. Freimuth, A. Kronenberg, J. Henrizi, I. Radu, E. Beaurepaire, Y. Mokrousov, P. M. Oppeneer, M. Jourdan, G. Jakob, D. Turchinovich, L. M. Hayden, M. Wolf, M. Münzenberg, M. Kläui, and T. Kampfrath, Nat. Photonics 10(7), 483-488 (2016).

[117] K. Kato, K. Takano, Y. Tadokoro, and M. Nakajima, Optics Letters 41, 2125-2128 (2016).

[118] T. Matsui, S. Tomita, M. Asai, Y. Tadokoro, K. Takano, M. Nakajima, M. Hangyo, and H. Yanagi, Applied Physics A 122, 157 (2016).

[119] Y. M. Bahk, G. Ramakrishnan, J. Choi, H. Song, G. Choi, Y. H. Kim, K. J. Ahn, D. S. Kim, and P. C. M. Planken, ACS Nano 8, 9089-9096 (2014).

[120] P. A. Obraztsov, N. Kanda, K. Konishi, M. KuwataGonokami, S. V. Garnov, A. N. Obraztsov, and Y. P. Svirko, Phys. Rev. B 90, 241416(R) (2014).

[121] O. V. Kibis, M. R. da Costa, and M. E. Portnoi, Nano Lett. 7, 3414-3417 (2007).

[122] L. V. Titova, C. L. Pint, Q. Zhang, R. H. Hauge, J. Kono, and F. A. Hegmann, Nano Lett. 15, 3267-3272 (2015).

[123] L. G. Zhu, B. Kubera, K. F. Mak, and J. Shan, Scientific Reports 5, 10308 (2014).

[124] B. S. Williams, Nat. Photonics 1(9), 517-525 (2007).

[125] A. Valavanis, J. Zhu, J. Freeman, L. Li, L. Chen, A. Davies, E. Linfield, and P. Dean, Electron. Lett. 50(4), 309-311 (2014).

[126] F. Wang, K. Maussang, S. Moumdji, R. Colombelli, J. R. Freeman, I. Kundu, L. Li, E. H. Linfield, A. G. Davies, J. Mangeney, J. Tignon, and S. S. Dhillon, Optica 2(11), 944 (2015).

[127] Q. Lu, D. Wu, S. Sengupta, S. Slivken, and M. Razeghi, Sci. Rep. 6, 23595 (2016). 
[128] S. Slivken and M. Razeghi, Proc. of SPIE 9755, 97550C (2016).

[129] A. Gorodetsky, N. Bazieva, and E. U. Rafailov, Quantumdot based ultrafast photoconductive antennae for efficient thz radiation, in: SPIE Proc., (2016), p. 97370C.

[130] H. Sherry, R. Al Hadi, J. Grzyb, E. Öjefors, A. Cathelin, A. Kaiser, and U.R. Pfeiffer, Lens-integrated thz imaging arrays in $65 \mathrm{~nm}$ cmos technologies, in: Radio Frequency Integrated Circuits Symposium (RFIC), 2011 IEEE, (2011), pp. 1-4.

[131] J. Van Rudd and D. M. Mittleman, JOSA B 19(2), 319329 (2002).

[132] D. Grischkowsky, S. Keiding, M. van Exter, and C. Fattinger, J. Opt. Soc. Am. B 7(10), 2006-2015 (1990).

[133] I. S. Gregory, C. Baker, W. R. Tribe, I. V. Bradley, M. J. Evans, E. H. Linfield, G. Davies, and M. Missous, Quantum Electronics, IEEE Journal of 41(5), 717-728 (2005).

[134] V. L. Malevich, Tech. Phys. Lett. 29(3), 240-242 (2003).

[135] M. M. Nazarov, A. P. Shkurinov, A. A. Angeluts, and D. A. Sapozhnikov, Radiophys. Quantum Electron. 52(8), 536545 (2010).

[136] X. C. Zhang, Y. Jin, T. Hewitt, T. Sangsiri, L. Kingsley, and M. Weiner, Applied Physics Letters 62, 2003-2005 (1993).

[137] V. Bespalov, V. Krylov, S. Putilin, and D. Staselko, Optics and Spectroscopy 93(1), 148-152 (2002).

[138] X. C. Zhang and D. H. Auston, J. Appl. Phys. 71(1), 326 (1992).

[139] D. H. Auston, K. P. Cheung, and P. R. Smith, Appl. Phys. Lett. 45(3), 284-286 (1984).

[140] Q. Chang, D. Yang, and L. Wang, Broadband thz generation from photoconductive antenna, in: Progress in electromagnetics research symposium, (2005), pp. 331-335.

[141] C. W. Berry and M. Jarrahi, Journal of Infrared, Millimeter, and Terahertz Waves 33(12), 1182-1189 (2012).

[142] S. E. Ralph and D. Grischkowsky, Applied Physics Letters 59(16), 1972-1974 (1991).

[143] E. R. Brown, F. W. Smith, and K. A. McIntosh, J. Appl. Phys. 73(3), 1480-1484 (1993).

[144] K. McIntosh, E. Brown, K. Nichols, O. McMahon, W. DiNatale, and T. Lyszczarz, Applied Physics Letters 67(26), 3844-3846 (1995).

[145] Y. S. Lee, Principles of Terahertz Science and Technology (Springer US, Boston, MA, 2009).

[146] C. A. Balanis, Antenna Theory: Analysis and Design, 3rd editio edition (Wiley-Blackwell, 2005).

[147] S. M. Duffy, S. Verghese, K. McIntosh, A. Jackson, A. Gossard, and S. Matsuura, Microwave Theory and Techniques, IEEE Transactions on 49(6), 1032-1038 (2001).

[148] E. Brown, International Journal of High Speed Electronics and Systems 13(02), 497-545 (2003).

[149] R. Mendis, C. Sydlo, J. Sigmund, M. Feiginov, P. Meissner, and H. Hartnagel, Antennas and Wireless Propagation Letters, IEEE 4, 85-88 (2005).

[150] M. Jarrahi, High-efficiency terahertz sources based on plasmonic contact electrodes, in: 2015 IEEE MTT-S Int. Microw. Symp., (IEEE, may 2015), pp. 1-4.

[151] P. Maraghechi and A. Elezzabi, Optics Express 18(26), 27336-27345 (2010).

[152] G. M. Rebeiz, Proceedings of the IEEE 80(11), 1748-1770 (1992).

[153] E. Brown, A. Lee, B. Navi, and J. Bjarnason, Microwave and optical technology letters 48(3), 524-529 (2006).
[154] S. Matsuura, M. Tani, and K. Sakai, Applied Physics Letters 70(5), 559-561 (1997).

[155] M. Tani, S. Matsuura, K. Sakai, and S. i. Nakashima, Applied optics 36(30), 7853-7859 (1997).

[156] K. Ezdi, M. Islam, Y. Reddy, C. Jördens, A. Enders, and M. Koch, A numerical study of photoconductive dipole antennas: the real emission frequency and an improved antenna design, in: Photonics Europe, (2006), p. 61940G.

[157] S. Verghese, K. McIntosh, and E. Brown, Microwave Theory and Techniques, IEEE Transactions on 45(8), 13011309 (1997).

[158] I. Gregory, W. Tribe, B. Cole, M. Evans, E. Linfield, A. Davies, and M. Missous, Applied Physics Letters 85(9), 1622-1624 (2004).

[159] T. K. Nguyen, H. Han, and I. Park, Journal of Infrared, Millimeter, and Terahertz Waves 32(6), 763-777 (2011).

[160] S. Lal, S. Link, and N. J. Halas, Nature Photonics 1, 641648 (2007)

[161] V. Giannini, A. I. Fernandez-Dominguez, S. C. Heck, and S. A. Maier, Chem. Rev. 111, 3888-3912 (2011).

[162] M.L. Brongersma and P.G. Kik, Surface plasmon nanophotonics (Springer, 2007).

[163] J. Shah, Hot Carriers in Semiconductor Nanostructures (Elsevier Inc., 1992).

[164] S. V. Makarov, V. A. Milichko, I. S. Mukhin, I. I. Shishkin, D. A. Zuev, A. M. Mozharov, A. E. Krasnok, and P. A. Belov, Laser and Photonics Reviews 10, 91-99 (2016).

[165] S. G. Park, Y. Choi, Y. J. Oh, and K. H. Jeong, Optics express 20(23), 25530-25535 (2012).

[166] Y. J. Oh and K. H. Jeong, Advanced Materials 24(17), 2234-2237 (2012)

[167] K. W. Vogt and P. A. Kohl, Journal of Applied Physics 74(10), 6448-6450 (1993).

[168] H. Surdi, A. Singh, and S. Prabhu, Enhancement of terahertz emission using auge nano-patterns, in: Proceedings of the 2013 COMSOL Conference in Bangalore, (2013).

[169] A. Singh, S. Pal, H. Surdi, S. Prabhu, V. Nanal, and R. Pillay, Applied Physics Letters 104(6), 063501 (2014).

[170] T. A. Liu, M. Tani, M. Nakajima, M. Hangyo, and C. L. Pan, Applied physics letters 83(7), 1322-1324 (2003).

[171] I. C. Mayorga, M. Mikulics, A. Schmitz, P. Van der Wal, R. Gusten, M. Marso, P. Kordos, and H. Luth, An optimization of terahertz local oscillators based on lt-gaas technology, in: SPIE Astronomical Telescopes+ Instrumentation, (2004), pp. 537-547.

[172] R. Mendis, C. Sydlo, J. Sigmund, M. Feiginov, P. Meissner, and H. L. Hartnagel, Solid-State Electronics 48(10), 2041-2045 (2004).

[173] G. Seniutinas, G. Gervinskas, E. Constable, A. Krotkus, G. Molis, G. Valušis, R. Lewis, and S. Juodkazis, Thz photomixer with a 40nm-wide nanoelectrode gap on lowtemperature grown gaas, in: SPIE Micro+ Nano Materials, Devices, and Applications, (2013), p. 892322.

[174] N. Khiabani, Y. Huang, L. E. Garcia-Munoz, Y. C. Shen, and A. Rivera-Lavado, Terahertz Science and Technology, IEEE Transactions on 4(4), 501-508 (2014).

[175] M. D. Wissert, A. W. Schell, K. S. Ilin, M. Siegel, and H. J. Eisler, Nanotechnology 20(42), 425203 (2009).

[176] E. Michael, B. Vowinkel, R. Schieder, M. Mikulics, M. Marso, and P. Kordoš, Applied physics letters 86(11), 111120 (2005). 
[177] Y. Hou, J. Liu, M. Buchanan, A. S. Thorpe, P. Poole, H. Liu, K. Wu, S. Roorda, and X. Zhang, Nano-Micro Letters 4(1), 10-13 (2012).

[178] H. Tanoto, J. Teng, Q. Wu, M. Sun, Z. Chen, S. Maier, B. Wang, C. Chum, G. Si, A. Danner et al., Scientific reports 3, 2824 (2013).

[179] M. Seo, H. Park, S. Koo, D. Park, J. Kang, O. Suwal, S. Choi, P. Planken, G. Park, N. Park et al., Nature Photonics 3(3), 152-156 (2009).

[180] B. Heshmat, M. Masnadi-Shirazi, R. B. Lewis, J. Zhang, T. Tiedje, R. Gordon, and T. E. Darcie, Adv. Opt. Mater. 1(10), 714-719 (2013).

[181] B. Heshmat, H. Pahlevaninezhad, Y. Pang, M. MasnadiShirazi, R. Burton Lewis, T. Tiedje, R. Gordon, and T. E. Darcie, Nano Lett. 12(12), 6255-9 (2012).

[182] A. Jooshesh, V. Bahrami-Yekta, J. Zhang, T. Tiedje, T.E. Darcie, and R. Gordon, Nano Lett. 15(12), 8306-8310 (2015).

[183] C. W. Berry and M. Jarrahi, Plasmonically-enhanced localization of light into photoconductive antennas, in: Conference on Lasers and Electro-Optics, (2010), p. CFI2.

[184] A. Krotkus, A. Arlauskas, and R. Adomaviius, Semiconductor investigation by terahertz radiation pulses (2012), pp. 84960V-84960V-10.

[185] P. B. Catrysse, G. Veronis, H. Shin, J. T. Shen, and S. Fan, Applied Physics Letters 88(3), 031101 (2006).

[186] B. Y. Hsieh and M. Jarrahi, Journal of Applied Physics 109(8), 084326 (2011).

[187] S. H. Yang and M. Jarrahi, Optics letters 38(18), $3677-$ 3679 (2013).

[188] I. S. Gregory, C. Baker, W. Tribe, M. Evans, H. E. Beere, E. H. Linfield, A. Davies, and M. Missous, Applied physics letters 83(20), 4199-4201 (2003).

[189] M. E. Fermann and I. Hartl, Nat. Photonics 7(11), 868-874 (2013).

[190] E. U. Rafailov, M. A. Cataluna, and W. Sibbett, Nat. Photonics 1(7), 395-401 (2007).

[191] M. A. Gaafar, A. Rahimi-Iman, K. A. Fedorova, W. Stolz, E. U. Rafailov, and M. Koch, Adv. Opt. Photonics 8(3), 370 (2016).

[192] K. Bertulis, A. Krotkus, G. Aleksejenko, V. Pacebutas, R. Adomavicius, G. Molis, and S. Marcinkevicius, Appl. Phys. Lett. 88(20), 201112 (2006).

[193] M. Suzuki and M. Tonouchi, Appl. Phys. Lett. 86(5), 051104 (2005).

[194] A. Krotkus and J. L. Coutaz, Semicond. Sci. Technol. 20(7), S142-S150 (2005).

[195] E. Estacio, M. H. Pham, S. Takatori, M. Cadatal-Raduban, T. Nakazato, T. Shimizu, N. Sarukura, A. Somintac, M. Defensor, F. C. B. Awitan, R. B. Jaculbia, A. Salvador, and A. Garcia, Appl. Phys. Lett. 94, 232104 (2009).

[196] A. E. Krasnok, D. Filonov, C. Simovski, Y. Kivshar, and P. Belov, Applied Physics Letters 104, 133502 (2014).

[197] A. B. Evlyukhin, S. M. Novikov, U. Zywietz, R. L. Eriksen, C. Reinhardt, S. I. Bozhevolnyi, and B. N. Chichkov, Nano Letters 12(7), 3749-3755 (2012).

[198] S. Makarov, S. I. Kudryashov, I. Mukhin, A. Mozharov, V. Milichko, A. Krasnok, and P. A. Belov, Nano Letters 15(9), 6187-6192 (2015).

[199] A. Krasnok, S. Makarov, M. Petrov, R. Savelev, P. Belov, and Y. Kivshar, SPIE Opt. and Optoelectr. 9502, 950203950203 (2015).
[200] A.E. Krasnok, A.E. Miroshnichenko, P. A. Belov, and Y. S. Kivshar, Opt. Express 20, 20599-20604 (2012).

[201] A. E. Krasnok, C. R. Simovski, P. A. Belov, and Y.S. Kivshar, Nanoscale 6, 7354-7361 (2014).

[202] R. M. Bakker, D. Permyakov, Y.F. Yu, D. Markovich, R. Paniagua-Dominguez, L. Gonzaga, A. Samusev, Y. S. Kivshar, B. Luk'yanchuk, and A. I. Kuznetsov, Nano Lett. 15, 2137-2142 (2015).

[203] R. Savelev, S. Makarov, A. Krasnok, and P. Belov, Optics and Spectroscopy (2015).

[204] P. A. Dmitriev, D. G. Baranov, V. A. Milichko, S. V. Makarov, I. S. Mukhin, A. K. Samusev, A. E. Krasnok, P. A. Belov, and Y. S. Kivshar, Nanoscale 8, 9721-9726 (2016).

[205] S. V. Makarov, A. Tsypkin, T. Voytova, V. Milichko, I. Mukhin, A. Yulin, S. Putilin, M. Baranov, A. Krasnok, I. A. Morozov, and P. Belov, Nanoscale DOI: 10.1039/C6NR04860A (2016).

[206] G. A. Wurtz, R. Pollard, W. Hendren, G. P. Wiederrecht, D. J. Gosztola, V. A. Podolskiy, and A. V. Zayats, Nature Nanotechnology 6(2), 107-111 (2011).

[207] V. K. Valev, D. Denkova, X. Zheng, A. I. Kuznetsov, C. Reinhardt, B. N. Chichkov, G. Tsutsumanova, E. J. Osley, V. Petkov, B. D. Clercq, A. V. Silhanek, Y. Jeyaram, V. Volskiy, P. A. Warburton, G. A. E. Vandenbosch, S. Russev, O. A. Aktsipetrov, M. Ameloot, V. V. Moshchalkov, and T. Verbiest, Advanced Materials 24, OP29-OP35 (2012).

[208] D. A. Zuev, S. V. Makarov, V. A. Milichko, S. V. Starikov, I. S. Mukhin, I. A. Morozov, I. I. Shishkin, A. E. Krasnok, and P. A. Belov, Adv. Mater. 28, 3087-3093 (2016).

[209] Y. Yang, W. Wang, A. Boulesbaa, I. I. Kravchenko, D. P. Briggs, A. Puretzky, D. Geohegan, and J. Valentine, Nano Lett. 15(11), 7388-7393 (2015). 